\title{
Biopolymers codelivering engineered T cells and STING agonists can eliminate heterogeneous tumors
}

\author{
Tyrel T. Smith, ${ }^{1}$ Howell F. Moffett, ${ }^{1}$ Sirkka B. Stephan, ${ }^{1}$ Cary F. Opel, ${ }^{2,3}$ Amy G. Dumigan, ${ }^{1}$ Xiuyun Jiang, ${ }^{4}$ Venu G. Pillarisetty, ${ }^{4}$ \\ Smitha P. S. Pillai, ${ }^{5}$ K. Dane Wittrup, ${ }^{2,3,6}$ and Matthias T. Stephan ${ }^{1,7,8}$ \\ ${ }^{1}$ Clinical Research Division, Fred Hutchinson Cancer Research Center, Seattle, Washington, USA. '2Department of Chemical Engineering, Massachusetts Institute of Technology (MIT), Cambridge, \\ Massachusetts, USA. ${ }^{3}$ Koch Institute for Integrative Cancer Research, MIT, Cambridge, Massachusetts, USA. ${ }^{4}$ Department of Surgery, University of Washington, Seattle, Washington, USA. ${ }^{5}$ Comparative \\ Pathology, Fred Hutchinson Cancer Research Center, Seattle, Washington, USA. ${ }^{6}$ Department of Biological Engineering, MIT, Cambridge, Massachusetts, USA. ${ }^{7}$ Department of Bioengineering and Molecular \\ Engineering and Sciences Institute, University of Washington, Seattle, Washington, USA. ${ }^{8}$ Department of Medicine, Division of Medical Oncology, University of Washington, Seattle, Washington, USA.
}

\begin{abstract}
Therapies using $T$ cells that are programmed to express chimeric antigen receptors (CAR T cells) consistently produce positive results in patients with hematologic malignancies. However, CAR T cell treatments are less effective in solid tumors for several reasons. First, lymphocytes do not efficiently target CAR T cells; second, solid tumors create an immunosuppressive microenvironment that inactivates $\mathrm{T}$ cell responses; and third, solid cancers are typified by phenotypic diversity and thus include cells that do not express proteins targeted by the engineered receptors, enabling the formation of escape variants that elude CAR T cell targeting. Here, we have tested implantable biopolymer devices that deliver CAR T cells directly to the surfaces of solid tumors, thereby exposing them to high concentrations of immune cells for a substantial time period. In immunocompetent orthotopic mouse models of pancreatic cancer and melanoma, we found that CAR T cells can migrate from biopolymer scaffolds and eradicate tumors more effectively than does systemic delivery of the same cells. We have also demonstrated that codelivery of stimulator of IFN genes (STING) agonists stimulates immune responses to eliminate tumor cells that are not recognized by the adoptively transferred lymphocytes. Thus, these devices may improve the effectiveness of CAR T cell therapy in solid tumors and help protect against the emergence of escape variants.
\end{abstract}

\section{Introduction}

Frontline cancer treatments like radiation and chemotherapy systemically interrupt critical cell processes and thus produce serious side effects, so there is considerable interest in developing immune-based treatments that can recognize and destroy tumor cells without harming healthy ones (1). For example, vaccines can be used to train the immune system to selectively destroy cancer cells. Unfortunately, the responses vaccines elicit may require months to mature and are usually insufficient to control advanced disease (2-5). In an effort to increase vaccine potency, various adjuvant compounds that stimulate tumor antigen uptake and cross-priming of naive $\mathrm{T}$ cells have been developed, and an understanding of innate signaling mechanisms has made it possible to rationally design more precise immunostimulants for treating cancer. One approach involves components of the stimulator of IFN genes (STING) pathway, which plays an important role in the detection of tumor cells by the immune system (6). In preclinical studies, cyclic nucleotides that activate this pathway have been shown to promote an aggressive antitumor response $(7,8)$. However, like previously described adjuvant compounds (e.g., R848 and related imidazoquinoline TLR7/8 agonists, muramyl dipeptides that trigger NOD-like receptors, and RNA oligonucleotide ligands

Authorship note: T.T. Smith, H.F. Moffett, and S.B. Stephan contributed equally to this work. Conflict of interest: The authors have declared that no conflict of interest exists.

Submitted: May 4, 2016; Accepted: February 23, 2017

Reference information: / Clin Invest. 2017;127(6):2176-2191.

https://doi.org/10.1172/JCl87624. of retinoic acid-inducible gene I [RIG-I]) (9-11), treatments using unformulated cyclic nucleotides are accompanied by systemic inflammatory toxicity, which creates a major hurdle for the implementation of these compounds to treat cancer patients $(12,13)$.

Another problem that impedes immunotherapeutic approaches is that endogenous $\mathrm{T}$ cell receptors (TCRs) typically have a low affinity for self/tumor antigens. To address this, an emerging cancer treatment strives to program patient-derived lymphocytes with genes encoding chimeric antigen receptors (CARs) that have been engineered to strongly bind proteins expressed by tumors $(14,15)$. It is also possible to cointroduce genes that can generate stimulatory signals to elicit robust $\mathrm{T}$ cell expansion, and, so far, CAR T lymphocyte therapy for hematological malignancies has produced impressive results in clinical trials $(16,17)$. The method involves retrieval of $\mathrm{T}$ cells from the patient and redirecting them ex vivo to express CARs composed of a tumor-specific single-chain antibody (scFv) fused to costimulatory and $\mathrm{CD} 3 \zeta$ signaling domains. These proteins enable the programmed cells to lyse tumor targets in an HLA-independent fashion. Unfortunately, this treatment has not proved very useful for treating solid tumors, because these cancers produce immunosuppressive signals that impede T cells (18), and because there is substantial phenotypic diversity in the proteins expressed by solid malignancies (Figure 1). Consequently, many cancer cells in solid tumors escape detection by the targeting CARs.

To overcome the problem of tumor-induced immune suppression, clinicians have used checkpoint antagonists to propel antitumor activities of programmed lymphocytes after they are returned to the patient (19). But because their application is sys- 


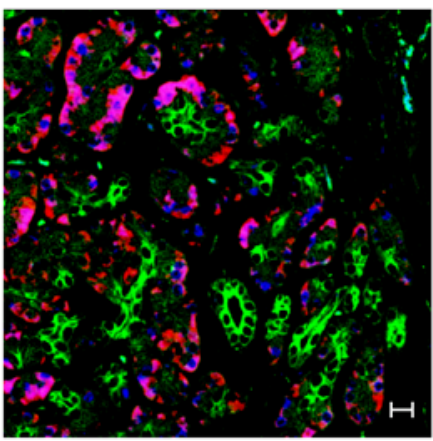

Pan-cytokeratin Mesothelin EpCAM

Figure 1. Solid tumors are heterogeneous and express diverse levels of antigens commonly used as targets for therapy. This representative confocal image of a human PDA shows the substantial diversity of proteins expressed by these tumors. Cytokeratin expression commonly occurs in adenocarcinomas, so we used a pan-cytokeratin antibody (green) to define tumor cell populations. The tumor differentiation antigen mesothelin (red) is a likely candidate for immunotherapy, and treatments targeting the cancer stem cell marker EpCAM (blue) are currently in clinical development. Scale bar: $100 \mu \mathrm{m}$. The image shown is representative of 20 randomly chosen fields.

temic, these treatments also disrupt immune homeostasis and induce dangerous autoimmune side effects (20-22). With regard to the problem of tumor heterogeneity, ideally, each patient should be treated with $\mathrm{T}$ cells that have been tailored to express CARs aligned to the tumor's protein fingerprint, which would protect these $\mathrm{T}$ cells from the emergence of antigen-loss variants. However, most clinics are not equipped or staffed to conduct the elaborate ex vivo procedures required to create a spectrum of targeted $\mathrm{T}$ cell variants for each cancer patient in their care. Furthermore, only a few CAR target antigens that are truly cancer specific have been identified, and systemically infusing more broadly targeted $\mathrm{T}$ cells can result in life-threatening "on-target/off-tumor" toxicities $(23,24)$.

Using biotechnology, we can address these problems by combining the benefits of CAR T cell therapy and cancer vaccines, while at the same time resolving some of their limitations. By doing so, we can provide clinicians with methods to pursue both local tumor destruction and systemic antitumor immunity. Specifically, we show here that appropriately engineered biomaterial delivery systems can efficiently deliver and functionally support tumor-targeting CAR T cells (see ref. 25 for an example) and at the same time chemically stimulate host immune responses to eliminate tumor cells that escape recognition by the adoptively transferred lymphocytes. Here, we use orthotopic models of inoperable pancreatic cancer and incompletely resected melanoma to demonstrate that biomaterial-mediated $\mathrm{T}$ cell delivery induces tumor regression more effectively than do systemic lymphocyte injections. We also establish that, beyond their primary function as $\mathrm{T}$ cell delivery vehicles, biomaterial implants can play a second important role: by releasing a vaccine adjuvant (such as the STING agonist cyclic di-GMP [cdGMP] tested here), these implants can convert the tumor into a "self" vaccine site, where eradicated cancer cells serve as sources of antigens that can launch an antitumor immune response by the host. This "second- wave" response is broader and is produced by a spectrum of $\mathrm{T}$ cells that can eliminate tumor variants not recognized by the programmed CAR T cells.

\section{Results}

Systemic injections of tumor-reactive $T$ cells do not eliminate pancreatic ductal adenocarcinoma. We used a cell line derived from the spontaneous pancreatic tumors produced by LSL-Kras ${ }^{G 12 D} p 53^{f /+}$ mice (referred to hereafter as KPC mice) (26) as the basis for an immunocompetent, orthotopic murine model of pancreatic cancer that has rapid and predictable growth kinetics. We genetically tagged the KPC cells with luciferase, so we could use bioluminescence imaging to noninvasively quantify tumor burdens. When orthotopically transplanted into the pancreas of non-KPC littermate mice, these tumor cells reproducibly developed into lesions that mimic human pancreatic cancer in terms of genetic mutations, histologic appearance, and heterogeneity of antigen/target expression (Figure 2, A-C).

In our first experiments, we verified that i.v. injections of pancreatic cancer-specific lymphocytes fail to eradicate tumors in this model. To create these lymphocytes, we transduced mouse T cells with a retrovirus encoding a chimeric NK receptor (composed of NKG2D linked to the cytoplasmic signaling domain of CD3ל) (ref. 27 and Figure 2D) that is specific for the KPC antigen ribonucleic acid export 1 (RAE1) (Figure 2, E and F). In order to track and quantify the in vivo migration of these cells in relationship to KPC tumors, we included vectors encoding click beetle red luciferase (CBR-luc) in the plasmid. Our results establish that, although these intravenously infused $\mathrm{T}$ cells accumulate in the spleen and liver, they inefficiently traffic to KPC tumor sites (Figure $2 \mathrm{G}$ ) and confer only a 4-day survival advantage compared with what was observed in the untreated controls (Figure 2H). Furthermore, expression levels of the RAE1 antigen were only slightly impaired by infusions of CAR-expressing $\mathrm{T}$ cells compared with levels detected with infusions of control lymphocytes (Figure 2I).

Delivery via bioactive carriers substantially improves $T$ cell expansion and function at the tumor site, but antigen-negative tumor subtypes escape elimination by them. We next explored the possibility of using biomaterials to mediate the localized delivery of tumor-reactive $\mathrm{T}$ cells at pancreatic tumor sites and to create ways to sustain these $\mathrm{T}$ cells. As described previously (25), our group recently developed a method to embed cancer-fighting immune cells into a resorbable polymeric device that can be surgically implanted where a tumor has been excised, or on the surface of one that is nonresectable (Figure 3A). After being positioned at their target site, the anticancer lymphocytes can begin eliminating tumor cells immediately. We produce these porous scaffolds from polymerized alginate, a castable, naturally occurring polysaccharide that has been approved by the FDA for human use because of its exceptional biocompatibility and biodegradability (28). To enable these scaffolds to function as efficient delivery vehicles for active $\mathrm{T}$ cells, we manufactured them with migration-promoting macromolecules (e.g., a collagenmimetic peptide) and stimulatory cues (e.g., microparticles displaying anti-CD3, anti-CD28, and anti-CD137 antibodies) (Figure 3A).

For these experiments, 10 days after infecting test mice with luciferase-expressing KPC tumor cells, we implanted scaffolds containing $7 \times 10^{6} \mathrm{NKG} 2 \mathrm{D}-\mathrm{CAR}^{+} \mathrm{T}$ cells directly onto the resulting 
A

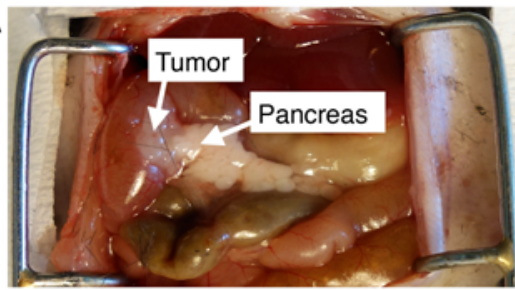

D

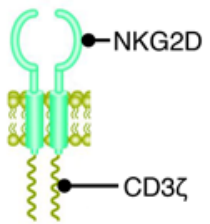

B
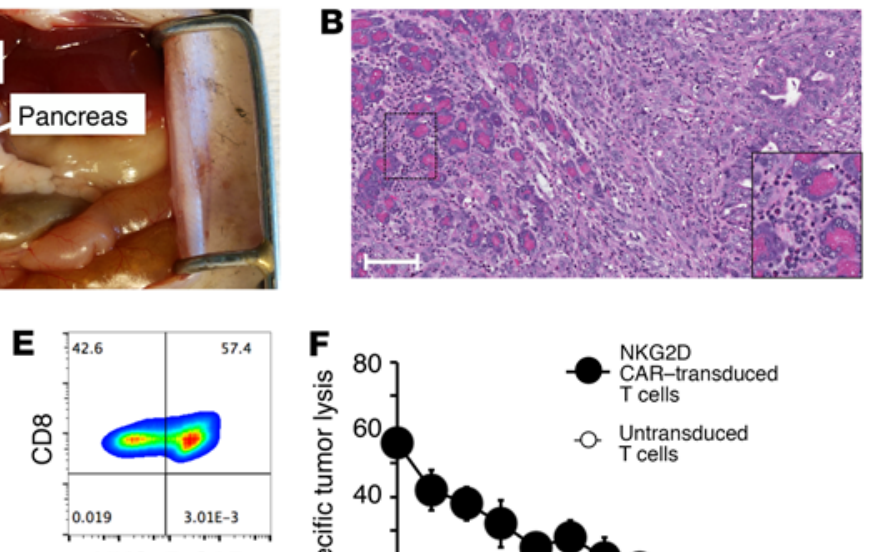

NKG2D CAR
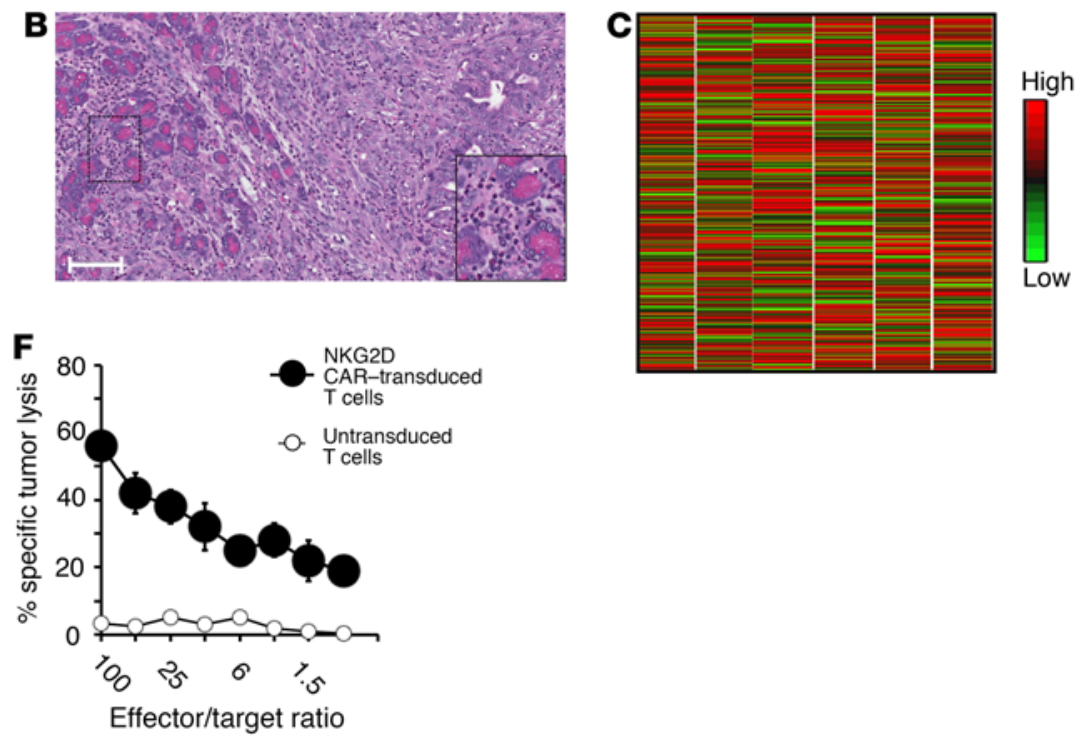

H

G

Treatment condition

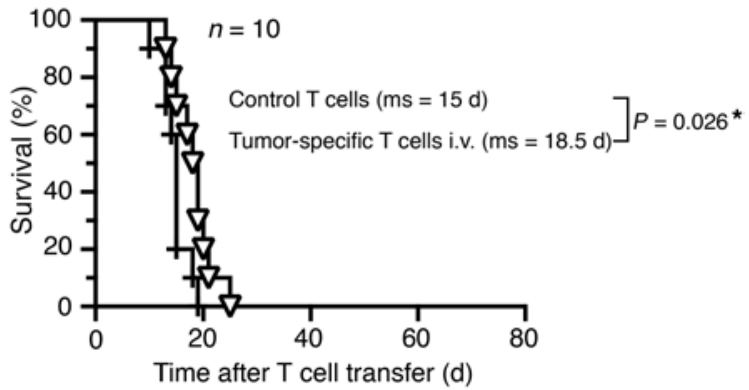

T cell imaging

Day 0

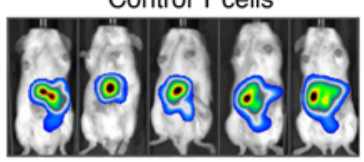
Tumor-specific T cells i.v.

Tumor imaging
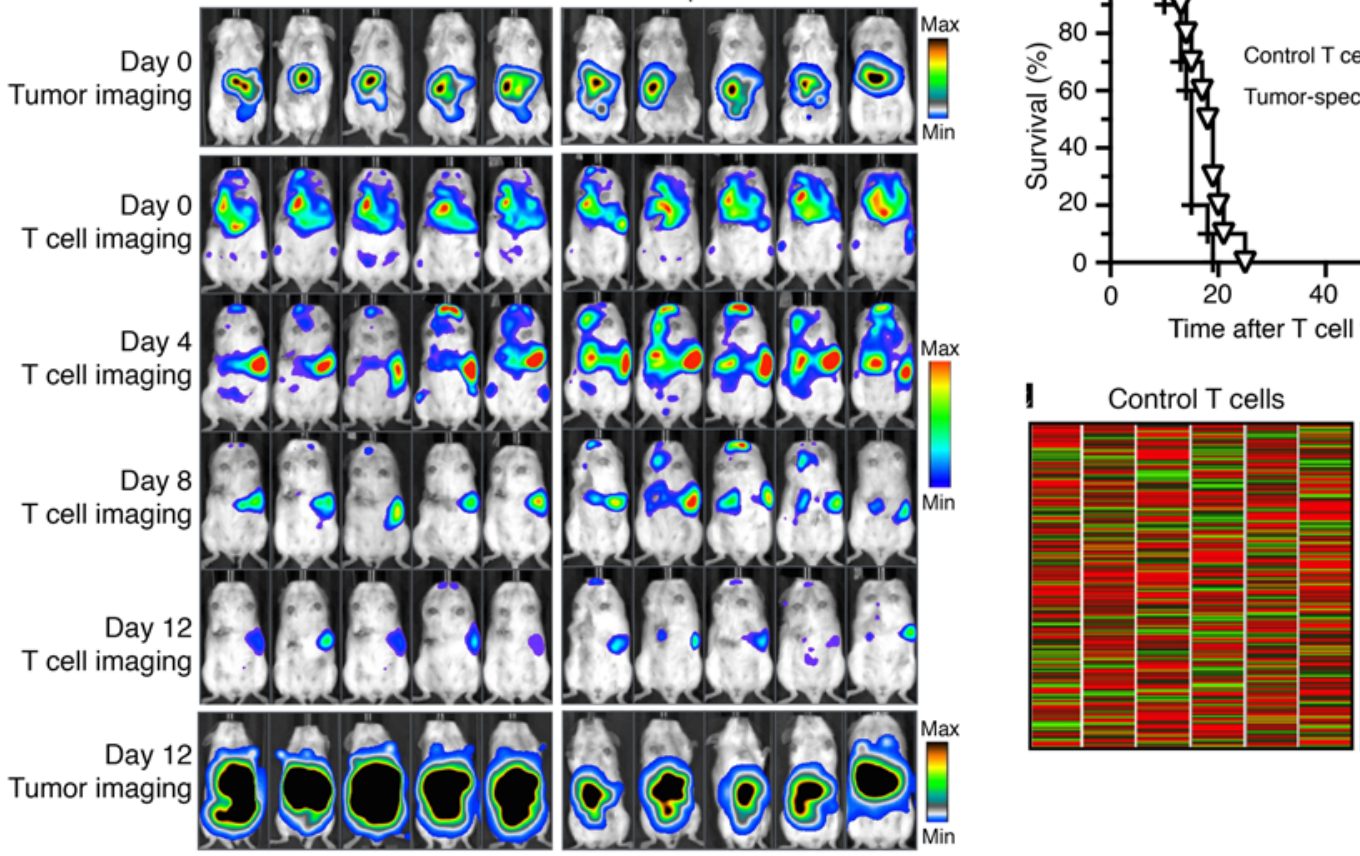

Tumor-specific T cells i.v.

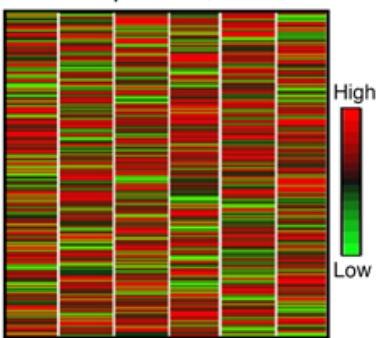

Figure 2. Systemic infusions of tumor-specific CAR T cells produce only modest therapeutic benefits. (A) Orthotopic mouse model of PDA. (B) Microscopy of KPC pancreas 7 days after implantation revealed invasive adenocarcinoma interspersed with parenchymal tissue; the adjacent healthy tissue accentuates the disorganized nature of the neoplastic regions. The formation of glands and ducts, hallmarks of adenocarcinoma, is also clear. Magnified images (e.g., inset) revealed substantial recruitment of immune cells, including polymorphonuclear leukocytes, within the tumor microenvironment. Scale bar: 100 $\mu \mathrm{m}$. Original magnification: $\times 20$. (C) Heatmap representation of flow cytometric data showing variability in RAE1 expression by KPC tumor cells. Tumors (7 days old) were disaggregated and labeled with anti-RAE1; the colors indicate expression levels in 1,800 randomly chosen cells pooled from 5 tumors compared with an isotype control. (D) The chimeric receptor we used to recognize RAE1 consisted of full-length mouse NCK2D fused to a murine CD3 $\zeta$ intracellular signaling molecule. (E) Flow cytometry measuring surface expression of NKC2D CARs after retroviral transduction and 3 days of expansion in medium containing G418. The profile shown is representative of 8 independent experiments. (F) ${ }^{51} \mathrm{Cr}$ release cytotoxicity assay of NKG2D CAR-transduced T cells reacting with KPC pancreatic tumor cells. Data are representative of 2 independent experiments. (G) Ten days after F-luc-expressing KPC tumor cells were transplanted into the pancreas, albino C57BL/6 mice were injected with $10^{7}$ NKC2D CAR-transduced T cells that coexpressed CBR-luc. Shown is sequential bioluminescence imaging of the tumors and T cells in 5 representative mice from each cohort $(n=10)$. (H) Kaplan-Meier survival curves for 10 mice per treatment group pooled from 3 independent experiments. ms, median survival. Statistical analysis of the treated experimental and the untreated control groups was performed using a log-rank test, and $P$ value of less than 0.05 was considered significant. Asterisks indicate statistical significance. (I) Heatmap representation of flow cytometric quantification of RAE1 antigen expression on KPC tumor cells following control or NKC2D CAR T cell therapy. Shown are 1,800 randomly chosen cells pooled from 5 tumors 
A

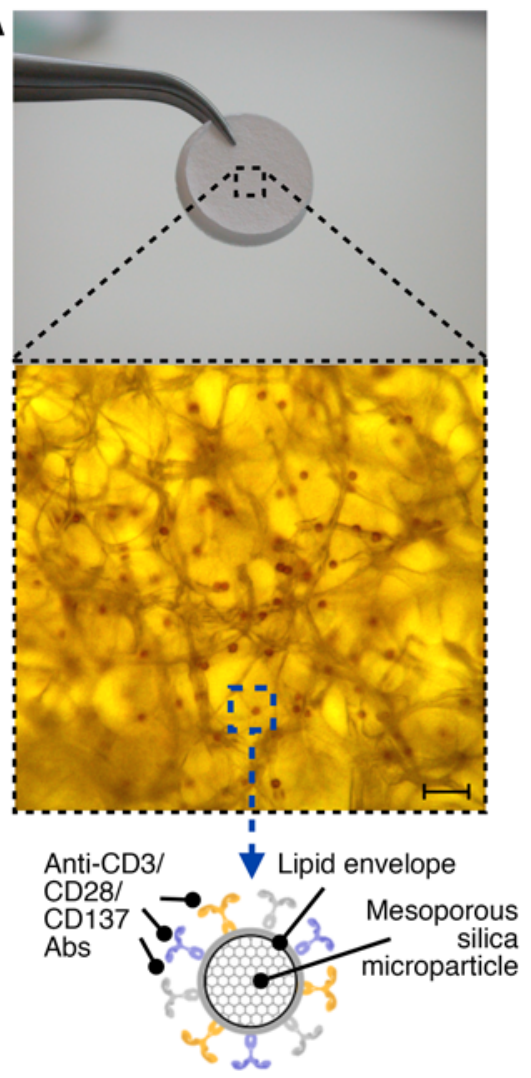

B
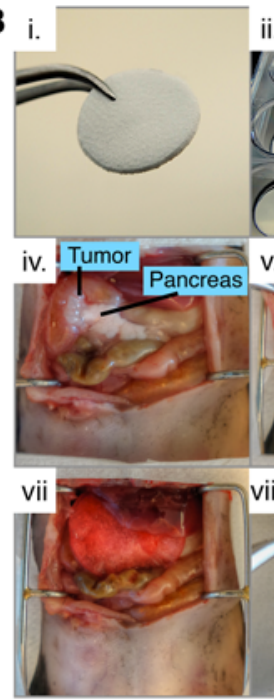

ii.

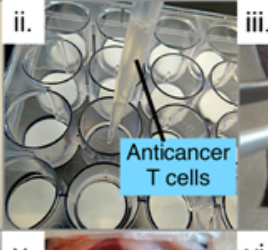

iii.
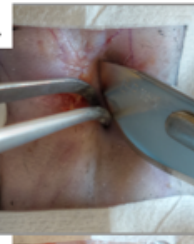

v.
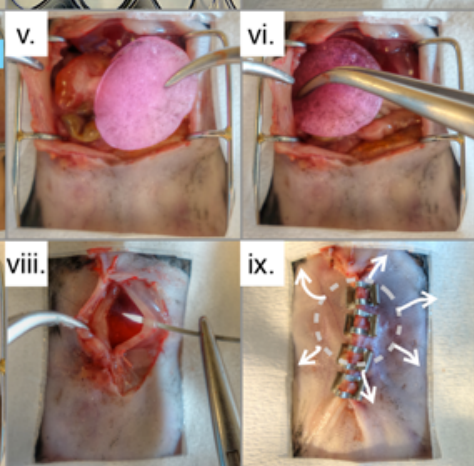

ix.

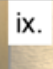

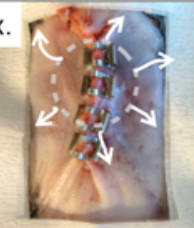

Figure 3. Biomatrices placed directly on pancreatic tumors provide an effective delivery platform for CAR-programmed T cells. (A) Bright-field microscopy of stimulatory microspheres incorporated into the biopolymer scaffold and a depiction of the composition of the microspheres. Scale bar: $70 \mu \mathrm{m}$. (B) This series of images illustrates our methodology: (i) biopolymer scaffold; (ii) seeding of tumorreactive T cells into the device; (iii) incision; (iv) orthotopic KPC pancreatic tumor; (v) implantation of a T cell-loaded device; (vi) wound closure; (vii-ix) sustained release of tumor-reactive T cells. pancreatic tumors (Figure 3B). A second group of mice received the same dose of cells injected directly into the tumors, and controls received no treatment. We used bioluminescence imaging to quantify tumor growth and (in parallel experiments) to track tissue distribution, expansion, and persistence of the lymphocytes. We found that the CAR T cells injected directly into pancreatic tumors persisted poorly in their immunosuppressive microenvironment and only produced a temporary delay in disease progression (median overall survival of 21 days versus 14.5 days in the untreated control group) (Figure 4, A-D). By contrast, T cells delivered from implanted polymer scaffolds underwent significant proliferation at the tumor site $(166$-fold higher peak photon count relative to injected T cells on day $8, P<0.0001$ ) (Figure 4 , $\mathrm{A}$ and $\mathrm{B}$ ) and substantially reduced KPC tumor growth (Figure 4, $A$ and $C$ ). However, even though they more than doubled the survival of treated mice, $\mathrm{T}$ cell-loaded scaffolds failed to completely clear the disease, and all mice eventually developed RAE1 low/ negative immune-escape variants (Figure 4E).

Combined delivery of CAR T cells and STING agonist by biomaterial implants results in a synergistic activation of host antigenpresenting cells. The results described above indicate that targeting single proteins with CAR T cells does not prevent the propagation of cancers via antigen-negative cells - even when the tumors are presented with high concentrations of anticancer lymphocytes delivered from a stimulating bioactive matrix. Accordingly, we next sought to simultaneously launch host $\mathrm{T}$ cell responses in an effort to eliminate residual CAR-resistant tumor cell types (Figure $5 \mathrm{~A}$ ). The immune system is able to generate effective cancerspecific responses, but it requires stimulation to do so. Unfortunately, tumors inhibit the maturation and activation of antigen- presenting cells (APCs) located in their draining lymph nodes and thereby prevent tumor-reactive $\mathrm{T}$ cells from differentiating into cytolytic effectors (18). In order to reverse this suppression, we used the biopolymer implants to provide high local concentrations of the immune-stimulatory STING agonist cyclic di-GMP (cdG$\mathrm{MP}$ ), thereby rendering the tumor milieu more conducive for $\mathrm{T}$ cell priming via the recruitment and stimulation of APCs (Figure $5 B)$. DCs, defined by their high expression of MHC II and T cell costimulatory molecules (e.g., CD86), are the most potent APCs and are capable of orchestrating an adaptive antitumor immune response. Immune phenotyping of tumor-associated lymph nodes associated with established KPC tumors revealed that less than $6 \%$ of their DCs (recognized in flow cytometry as CD11 $\mathrm{c}^{+} \mathrm{CD} 11 \mathrm{~b}^{+}$) were appropriately activated, as evidenced by their expression of CD86; the majority of the DCs were tolerogenic (CD86-) (Figure 6A). Releasing only cdGMP from implanted scaffolds upregulated CD86 and MHC II expression by a large proportion of these DCs and increased their overall frequency by 38 -fold in the draining lymph nodes (Figure 6B). Following implantation of CAR T cellloaded scaffolds fabricated without the STING agonist, the numbers of $\mathrm{CD} 11 \mathrm{c}^{+} \mathrm{CD} 86^{+}$mature DCs increased to a lesser degree (9.4-fold), but the MHC II expression levels on these cells were more than twice as high compared with the those in the cdGMP treatment group (Figure 6, A and B), which was presumably the result of tumor antigen release by the cytolytic CAR T cells. The release of both cdGMP and CAR T cells from implanted matrices produced a synergistic activation of DCs, which was reflected by robust increases in the frequencies of activated DCs (3.7-fold higher compared with cdGMP alone). Notably, these cells expressed high levels of costimulatory molecules as well as MHC II, indi- 
A

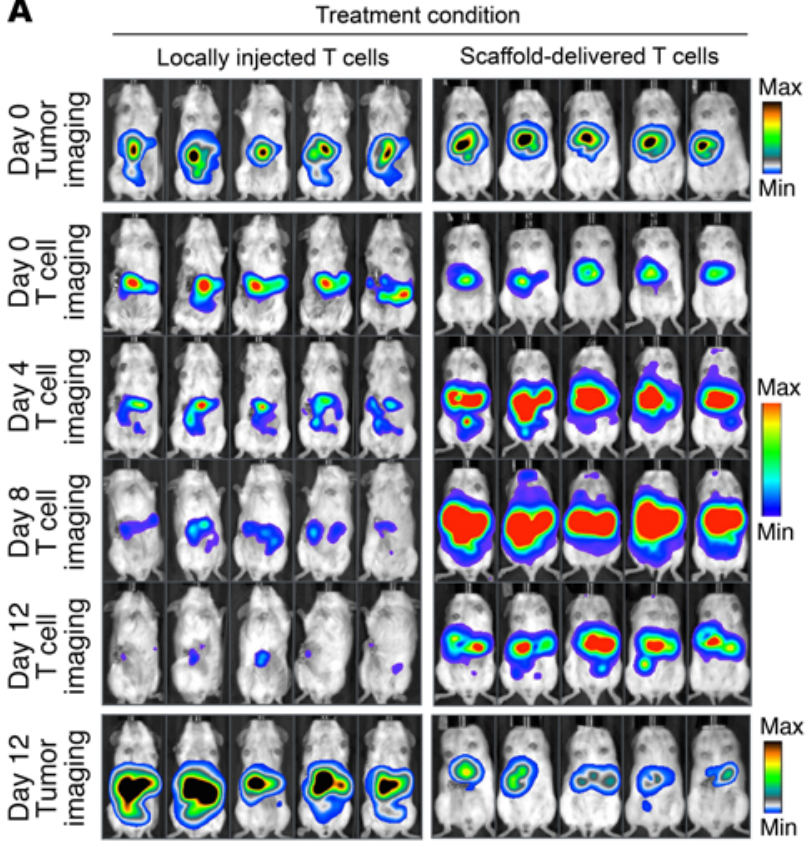

B

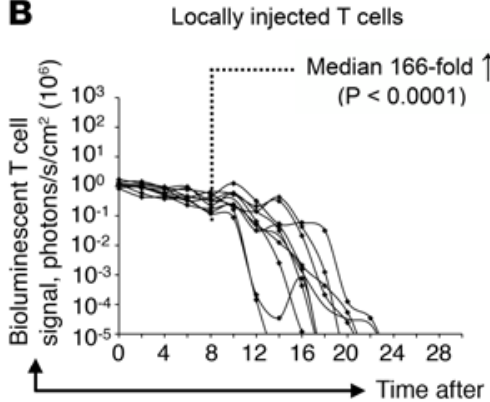

C

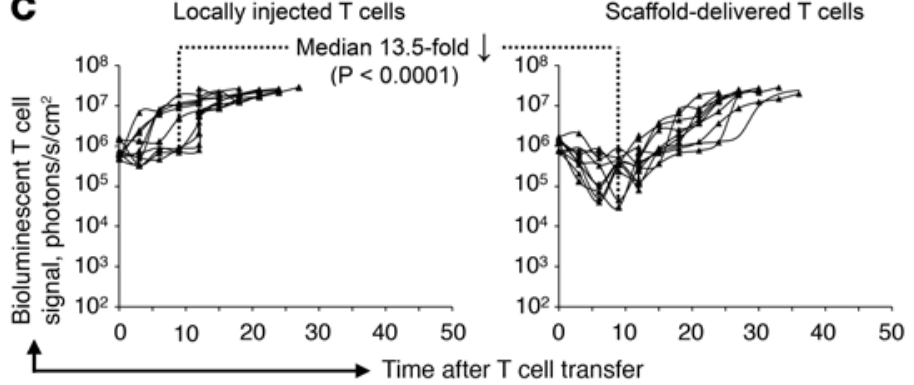

Scaffold-delivered T cells

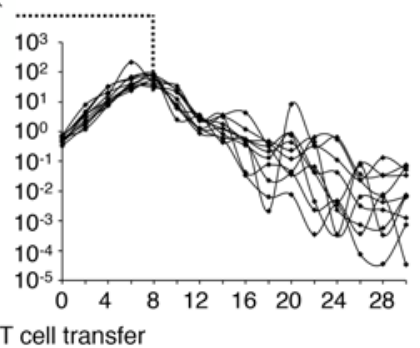

D

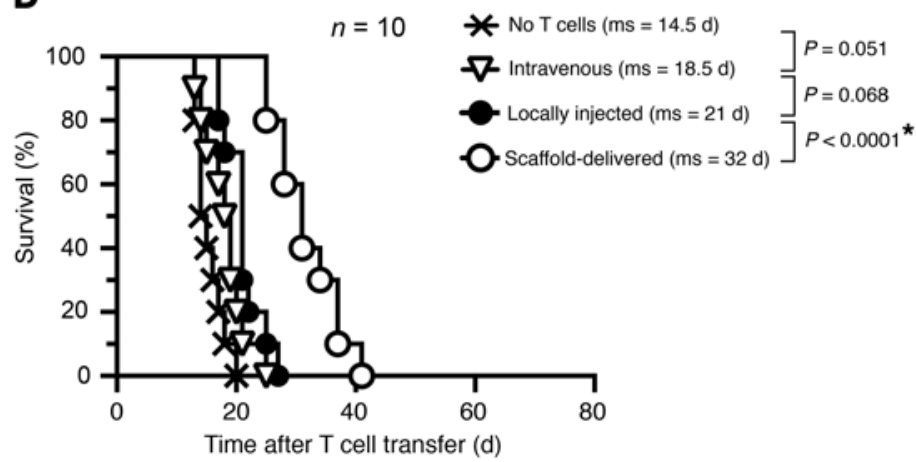

E

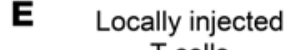
T cells

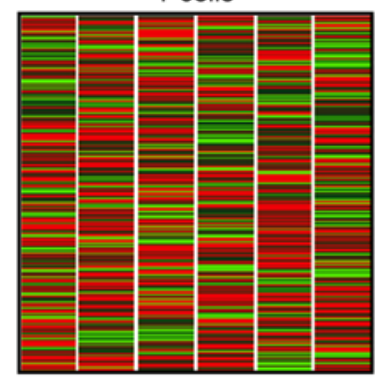

Figure 4. Polymer-launched CAR T cells robustly expand at the tumor site and bring about tumor regression, but spare cells that do not express the target antigen. (A) Bioluminescence imaging of KPC tumors and adoptively transferred CAR T cells. Mice were treated with $10^{7}$ NKC2D-transduced Iymphocytes injected locally into the tumor or contained in bioactive scaffolds implanted directly onto the tumor surface. Five representative mice from each cohort $(n=10)$ are shown. (B) T cell signal intensities from sequential bioluminescence images captured every 2 days after cell transfer. Each line represents 1 animal, and each dot denotes the whole-animal photon count. At the indicated time points, pairwise differences in photon counts between treatment groups were analyzed using the Wilcoxon rank-sum test. Shown are data for 10 mice per treatment condition pooled from 3 independent experiments. (C) Quantification of KPC bioluminescent tumor signal. Pairwise differences in the bioluminescent T cell signals were analyzed at selected time points using the Wilcoxon rank-sum test. A $P$ value less than 0.05 was considered significant. (D) Kaplan-Meier survival curves for treated and control mice. Shown are 10 mice per treatment group pooled from 3 independent experiments. Statistical analysis between the experimental and control groups was performed using the log-rank test, and a $P$ value of less than 0.05 was considered significant. Asterisks indicate statistical significance. (E) Flow cytometric quantification of RAE1 antigen expression on KPC tumor cells following NKG2D CAR T cell therapy. Shown are 1,800 randomly chosen cells pooled from 5 tumors.

cating that they can efficiently cross-present tumor antigens and launch antitumor $\mathrm{T}$ cell responses (Figure 6B).

Combined CAR $T$ cell and STING agonist therapy primes robust tumor-specific host lymphocyte responses. To directly visualize the location and magnitude of endogenous $\mathrm{T}$ cell activation following different treatment regimens, we implanted KPC tumors into transgenic mice that express nuclear factor of activated T cells (NFAT) tagged with a luciferase reporter (NFAT-luc mice). NFAT is a prominent transcription factor downstream of the TCR/CD3 signaling cascade. Stimulation of TCRs activates calcineurin, which dephosphorylates NFAT; within minutes, the dephosphorylated NFAT proteins translocate from the cytoplasm into the nucleus, where they regu- late genes for cytokines and other proteins that are critical for the immune response (29). The NFAT-luc-transgenic mice used here contain an NFAT response element that drives the transcription of the luciferase reporter gene, so they emit light following TCR activation. This process can be serially quantified using bioluminescence imaging. We treated mice bearing KPC pancreatic tumors with biomaterial scaffolds engineered to release either cdGMP, CAR T cells, or a combination of the two. Control mice received no treatment. We used bioluminescence imaging to monitor the NFAT-luc signal every 2 days, over a period of 30 days. No bioluminescence above background levels could be detected in untreated mice (Figure 7, A and B). However, mice receiving scaffolds functionalized either with 


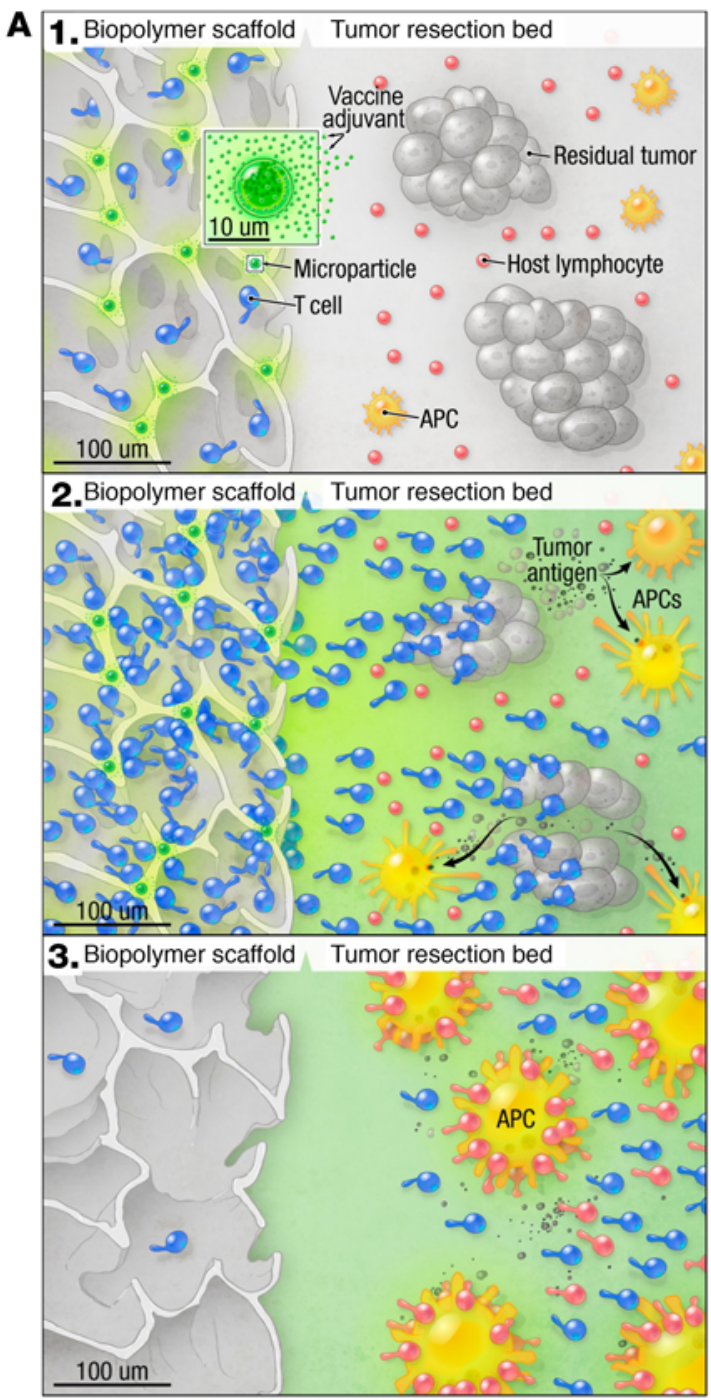

B
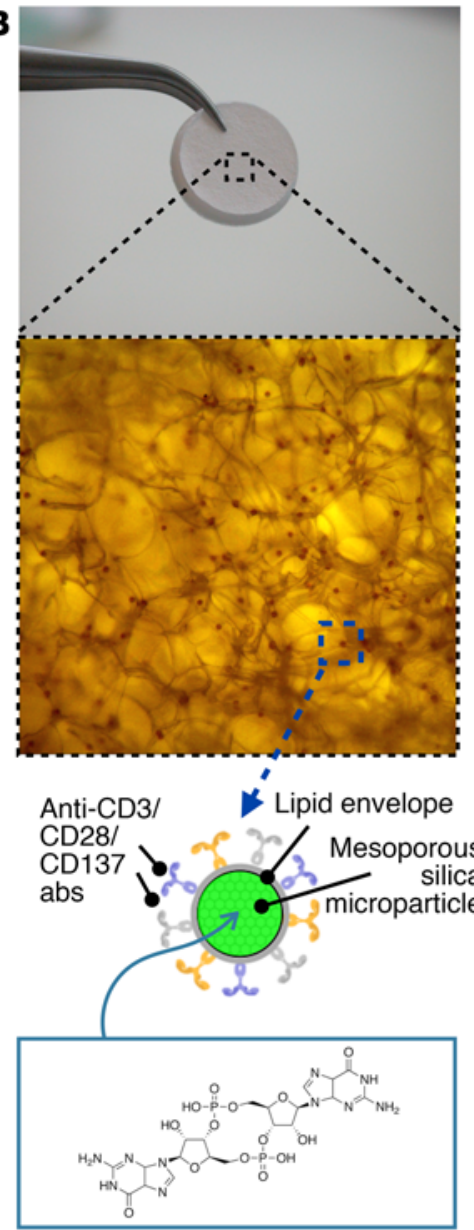

STING agonist cdGMP
Figure 5. Design of a biomaterial carrier that codelivers CAR-expressing $T$ cells and vaccine adjuvant to simultaneously clear heterogeneous cancer cells and establish systemic antitumor immunity. (A) Schematic diagrams of a scaffold loaded with CAR T cells interacting with the tumor bed: panels 1 and 2 show how factor-containing microspheres incorporated into the device stimulate the expansion of CAR-expressing $T$ cells and promote their egress into surrounding tissue. Panels 2 and 3 illustrate the release of vaccine adjuvant from $T$ cell-loaded implants, priming host immune cells to recognize and lyse tumor cells and thereby protect against antigen escape variants. (B) Macro- and microscopic views of a porous alginate matrix functionalized with microparticles that have the STING agonist cdGMP entrapped in the polymer core and stimulatory anti-CD3/CD28/CD137 antibodies tethered to their phospholipid membrane. Schematic shows the chemical structure of cdGMP.
cdGMP or CAR T cells showed luciferase activity in the tumor area, which peaked between days 8 and 10 following implantation of the devices. The combined release of cdGMP and T cells from implanted matrices triggered the maximal NFAT activation (5.6-fold higher peak photon count relative to that of T cells only on day $10, P<$ 0.0001; Figure 7, A and B). Notably, in this treatment group, luciferase signals extended well beyond the pancreatic tumor region into the spleen and mesenteric lymph nodes, indicating host $\mathrm{T}$ cell activation in these organs (Figure 7A, lowest panel).

To verify that the recorded bioluminescent NFAT signals accurately reflect activation of tumor-specific T cells in the host, the experiments were repeated using a pancreatic tumor model in which the KPC cells express the lymphocytic choriomeningitis virus (LCMV) glycoprotein GP33. As a surrogate pancreatic tumor antigen, this protein enabled us to use flow cytometry to analyze how the biopolymer implants affect the frequency of GP33specific lymphocytes among circulating $\mathrm{CD}^{+} \mathrm{T}$ cells. In order to distinguish between scaffold-delivered and endogenous $\mathrm{T}$ cells, we genetically tagged the donor cells with a CD45.2 marker and used CD 45.1- transgenic mice as hosts. We treated mice with biomaterial scaffolds engineered to release into the tumor either cdGMP, CAR T cells, or a combination of the two. The control mice received no treatment. As expected, spontaneous antitumor $\mathrm{T}$ cell responses rarely occurred in the untreated mice (Figure $7 \mathrm{C}$ ), establishing that, despite their expression of the GP33 xenoantigen, KPC-GP33 tumors continued to be highly immunosuppressive. Treatment with cdGMP-loaded scaffolds, or those delivering lymphocytes alone, produced only modest host antitumor $\mathrm{T}$ cell activities (1.3-fold and 2-fold increases in circulating GP33specific T cells, respectively) (Figure 7, C and D). By contrast, the combination of cdGMP and CAR-expressing T cells elicited synergistic antitumor responses by host cells, which were, on average, 6.4-fold higher than the responses of implants releasing only lymphocytes (Figure 7, C and D).

STING-delivering implants can trigger host antitumor immunity sufficient to clear tumors and eliminate metastases. To measure the antitumor benefits provided by biopolymer scaffolds that codeliver the STING agonist along with CAR-programmed T cells, we treated mice bearing orthotopic KPC tumors with scaffolds functionalized with either cdGMP alone or both cdGMP and anticancer CAR T cells. As a specificity control, a separate group of mice received scaffolds loaded with STING agonist and tumorirrelevant (anti-GP75) CAR T cells. To enable a side-by-side comparison of biomaterial-mediated delivery versus local injection, 
A

\section{A}
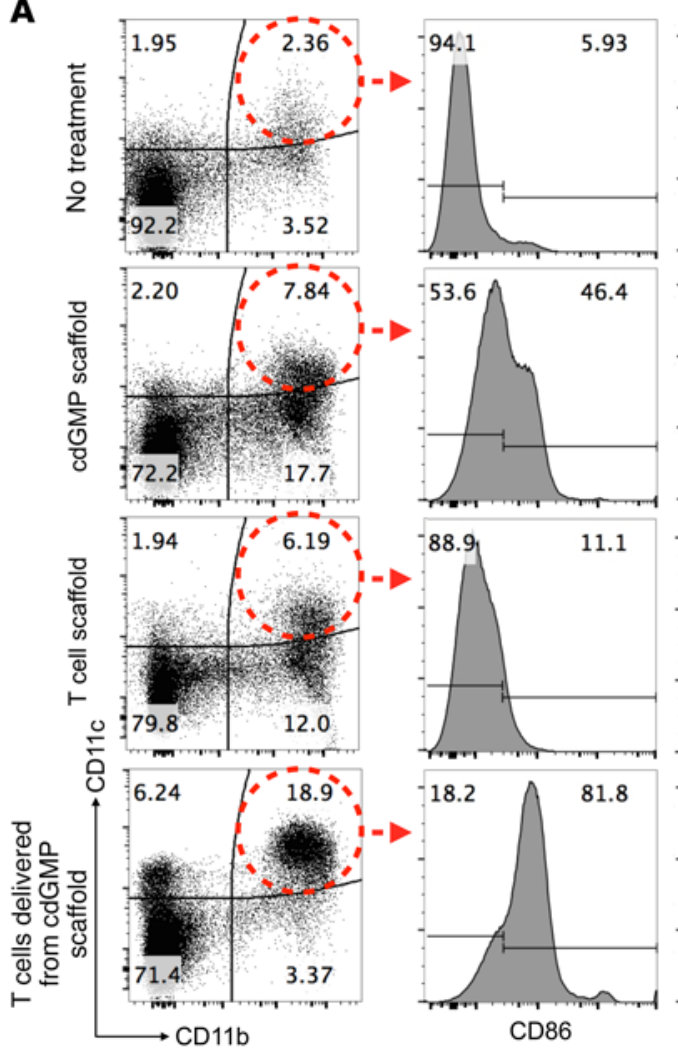

CD86
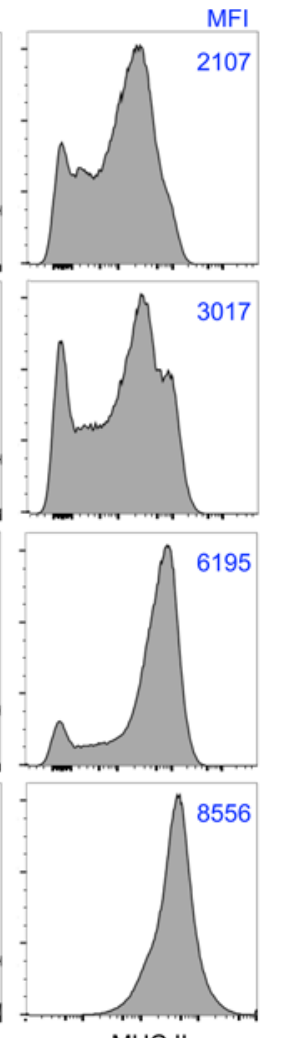

$\mathrm{MHC} \|$

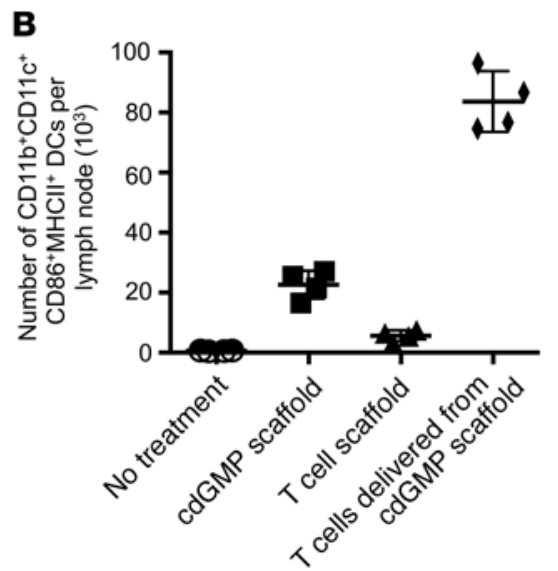

Figure 6. Scaffold-released CAR T cells and STING agonist synergize to activate host APCs. (A and B) Ten days after transplanting luciferase-expressing KPC tumor cells into the pancreases of mice, we implanted scaffolds containing either $7 \times 10^{6}$ tumor-reactive CAR T cells, $6 \mu$ g cdGMP, or a combination of both, onto the tumor surface. The control mice received no treatment. Five days later, peripancreatic lymph nodes were digested into cell suspensions for analysis by flow cytometry. We only tested lymph nodes that were not engulfed by tumors and pooled them from at least 5 animals. (A) Flow cytometric plots of myeloid maturation markers (CD11b and CD11c). Histograms depict the expression of the costimulatory factor CD86 and MHC II molecules after gating on $\mathrm{CD} 11 \mathrm{C}^{+} \mathrm{CD} 11 \mathrm{~b}^{+}$double-positive cell populations. The profiles shown here are representative of 4 independent experiments. MFI, mean fluorescence intensity. (B) Absolute numbers of mature and activated (CD11 $\mathrm{b}^{+} \mathrm{CD} 11 \mathrm{C}^{+} \mathrm{CD} 8 \mathrm{6}^{+} \mathrm{MHCII}$ ) DCs in peripancreatic lymph nodes. Points represent the number of cells per lymph node in samples pooled from 5 mice, and the data are representative of 4 separate experiments. Each bar represents the average number of cells \pm SD.

we also included 2 groups that were treated with an intratumor injection of cdGMP (with or without tumor-specific CAR T cells). The control mice received no treatment. Serial bioluminescence imaging of tumor growth confirmed that the combined release of CAR T cells and cdGMP from biomaterial implants eradicated KPC tumors in 4 of 10 treated mice (Figure 8 , A and B). The remaining 6 mice showed substantial tumor regression, with an average increase in survival of 37 days, which was a 4.6 -fold improved outcome compared with cointroducing CAR T cells and the STING agonist by injection into the tumors. Importantly, the synergistic antitumor effect was dependent on tumor-specific CAR $\mathrm{T}$ cells, as codelivering tumor-irrelevant anti-GP75 CAR $\mathrm{T}$ cells using STING agonist-functionalized scaffolds improved survival by only 5 days. To exclude the possibility that luciferase affected the T cell response we achieved using STING/CAR T cell scaffolds, we performed independent experiments that compared the survival rates of mice bearing unmodified versus luciferase-tagged KPC pancreatic tumors. Animals were treated with scaffolds that either codelivered the STING agonist along with CAR-programmed T cells, or they received no therapy. We found that luciferase expression had no significant effect on the thera- peutic outcome (Figure 8C), indicating that in our test system the luciferase protein is not a dominant neoantigen.

To determine whether the implants can elicit global antitumor immunity, we rechallenged the 4 mice that experienced complete tumor regression (Figure 9) with a systemic dose of $10^{4}$ luciferaseexpressing KPC tumor cells; tumor-naive mice were used as controls. We then used bioluminescence imaging to quantify differences in growth rates of lung metastases between the treatment groups. The results showed that all mice cured with combined CAR T cell and STING agonist immunotherapy were fully protected from this rechallenge, with no measurable tumor mass 4 weeks after the KPC cells were administered. By contrast, the control animals quickly formed metastatic foci in their lungs and rapidly succumbed to their disease, as would be expected (Figure 9). Thus, our evidence shows that appropriately formulated combination therapy using CAR-programmed T cells and STING agonists can eliminate local tumors and trigger systemic host antitumor immunity powerful enough to prevent untreated distant metastases.

We next measured the potential side effects the biomaterial implants may have on pancreatic function by monitoring the mice for weight loss and measuring their serum levels of pancreatic 
A

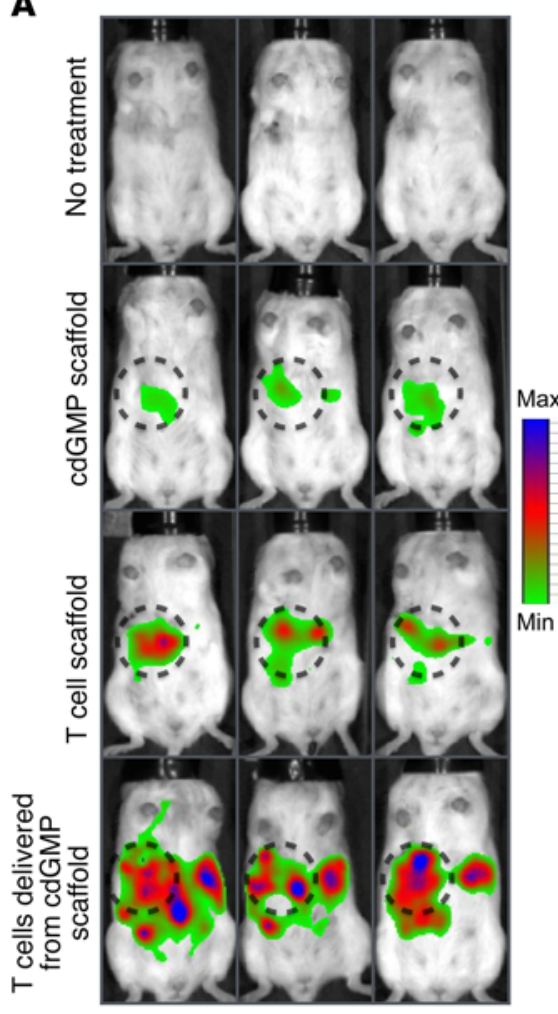

B

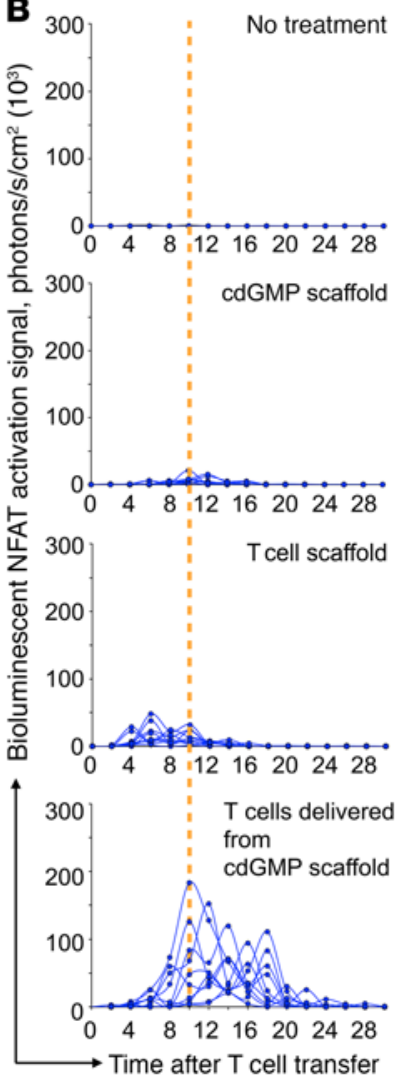

C

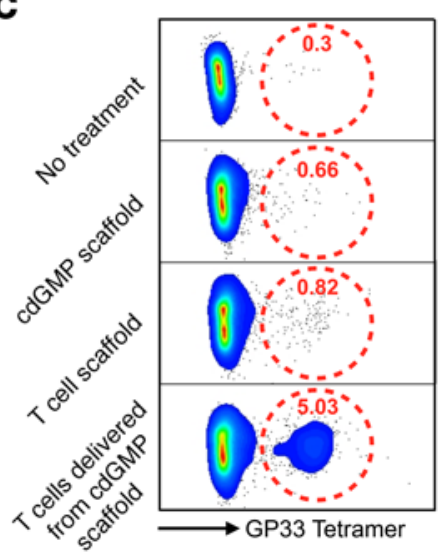

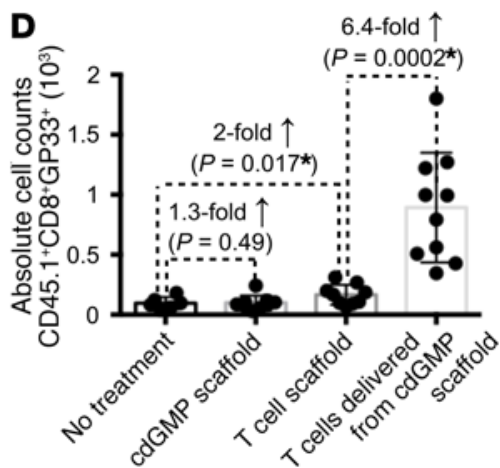

Figure 7. Corelease of cdGMP along with CAR-expressing T cells from biomaterial implants primes endogenous tumor-reactive lymphocytes. (A and B) KPC tumors were transplanted into NFAT-luc-expressing transgenic mice that had an albino background (which improves bioluminescence signal detection). We treated these mice with biomaterial-delivered cdGMP, CAR T cells, or a combination of the two, and monitored the NFAT-luc signal, which quantifies activated host T cells, every 2 days over a period of 30 days. (A) Bioluminescence imaging of 3 representative mice per treatment group 10 days after scaffold implantation (dashed circles indicate implantation sites). Shown are 3 representative mice from each cohort ( $n=10$ in 2 independent experiments). (B) NFAT-luc signal intensities after treatments. Each line represents 1 animal, and each dot reflects the whole-animal photon count. (C and D) To confirm that the bioluminescence NFAT-luc signals reflected endogenous tumor-reactive T cell counts, we treated mice that had KPC pancreatic tumors expressing GP33 and then quantified host GP33-specific T cells in the peripheral blood by tetramer staining. For these studies, congenic CD45.1 recipient mice were used to distinguish endogenous from adoptively transferred T cells. (C) Representative flow cytometric plots showing the percentages of GP33 tetramer-positive cells in peripheral blood 10 days after scaffold implantation, gated on CD45.1 $1^{+}$(host) CD8 $8^{+}$cells. (D) Absolute numbers of primed $\left(\mathrm{CD} 45.1^{+} \mathrm{CD}^{+} \mathrm{CP} 33^{+}\right) \mathrm{T}$ cells. Each point represents the number of cells in peripheral blood per mouse. Each bar represents the average cell count \pm SD. Shown are 10 mice pooled from 3 independent experiments. $P$ values obtained by two-tailed Student's $t$ test.

lipase and amylase 1 week after treatment. We found no significant difference in body weights among the different groups (Figure 8D), but we found that serum amylase levels in mice treated with STING agonist/CAR T cell implants were 1.3-fold lower than levels in the untreated tumor-bearing mice (average $640 \mathrm{U} / 1 \pm 17.9$ versus 826 $\mathrm{U} / 1 \pm 54.7 ; P=0.009 ;$ Figure $8 \mathrm{E}$ ). In mice, normal serum amylase activity consists mainly of salivary amylase, because pancreatic amylase is not resorbed by renal tubular epithelial cells and is rapidly excreted in the urine (30). This means that in mice, caution must be taken when amylase values are used to evaluate pancreatic function. We found that serum lipase activity was also lower in the mice that were treated with cdGMP/CAR T cell scaffolds compared with untreated tumor-bearing mice $(82 \mathrm{U} / \mathrm{l} \pm 4.5$ versus 128 $\mathrm{U} / 1 \pm 10.85 ; P=0.003)$. While a reference range for comparison of serum lipase activity in mice is not available, the reduced enzyme activity might indicate an exocrine pancreatic insufficiency due to the destruction or loss of acinar cells; however, a histological correlate for this was not found (Figure $8 \mathrm{~F}$, left panel), and 3 weeks after scaffold implantation, the pancreases in survivors that had rejected disease showed a normal histological appearance (Figure $8 \mathrm{~F}$, right panel), with no evidence of necrosis or inflammation.

Effects of CAR T cell and STING agonist therapy on melanoma cells. To confirm that these findings have relevance for other types of cancer, we created a second test system by subcutaneously injecting B16F10 melanoma cells into mice. Ten days later, we resected tumors but left behind $1 \%$ to $5 \%$ of the diseased tissue (Figure 10A). In all untreated animals, post-resection tumor regrowth was observed, and approximately $40 \%$ of these mice developed palpable lymph node metastases.

We chose the tyrosinase-related protein 1 (or GP75) antigen (31) as the therapeutic target for melanoma, because it is the most abundant glycoprotein synthesized by pigmented melanocytes and melanomas. The fact that GP75 is specific for melanocytes along with both primary and metastatic melanomas makes it a very attractive immunotherapeutic target to treat this disease $(32,33)$. However, in our system, the surface expression of GP75 by tumor 

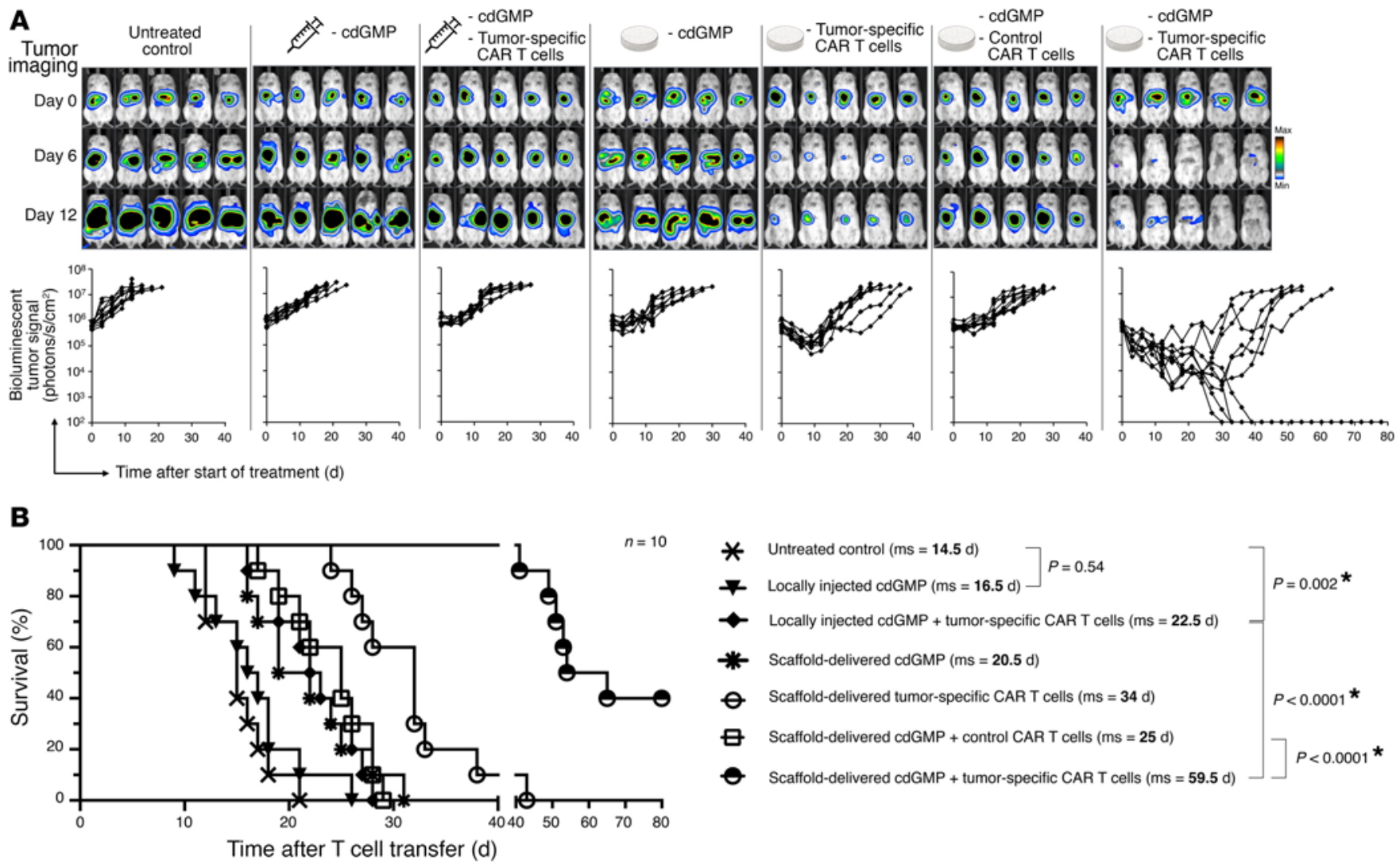

C

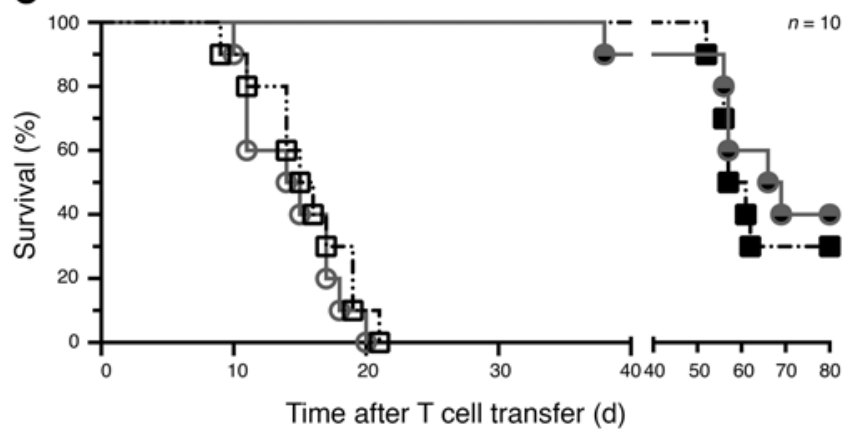

$\left.\begin{array}{ll}\ominus & \text { Untreated control, KPC }(\mathrm{ms}=14 \mathrm{~d}) \\ € & \text { Untreated control, } \mathrm{KPC} \text {-luc }(\mathrm{ms}=15 \mathrm{~d})\end{array}\right] P=0.46$

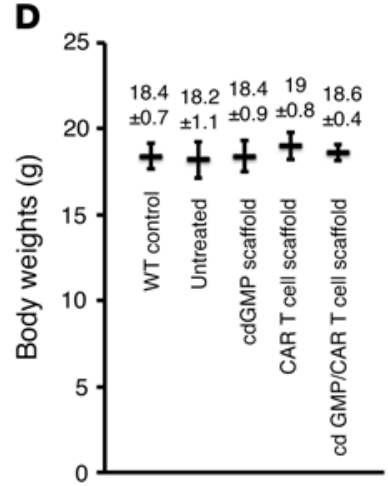

E

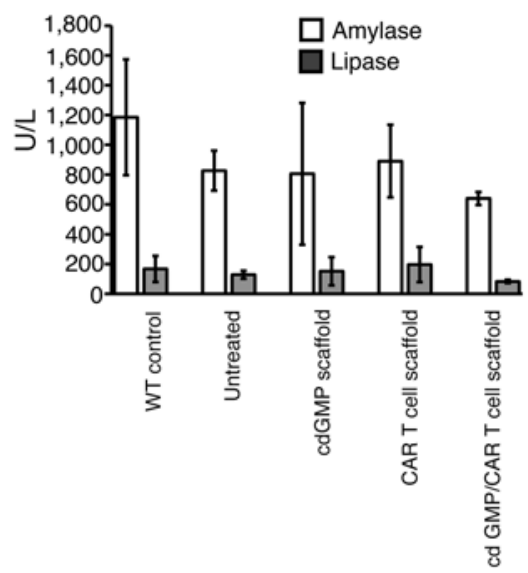

F

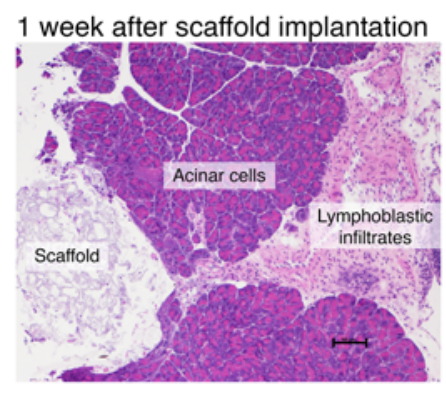

3 weeks after scaffold implantation 
Figure 8. Implants that codeliver STING agonists along with CAR-expressing T cells can limit tumor immune escape in established inoperable tumors. (A) Serial in vivo bioluminescence imaging of KPC-luc tumors. Shown are 5 representative mice from each cohort ( $n=10$ mice in 3 independent experiments). Quantification of KPC bioluminescent tumor signals is also shown. (B) Kaplan-Meier survival curves for treated and control mice. Statistical analysis of the treated experimental and the untreated control groups was performed using the log-rank test, and a $P$ value of less than 0.05 was considered significant. Asterisks indicate statistical significance. (C) Independent experiments showing survival and treatment responses of mice bearing unmodified versus luciferase-tagged KPC pancreatic tumors. Data obtained using the Log-rank test. (D-F) Assessment of side effects biomaterial implants have on pancreatic functions. (D) Average weight changes ( \pm SD) compared with control mice weights 1 week after treatment. Shown are 10 mice pooled from 2 independent experiments. (E) Serum levels of amylase and lipase. Each bar represents the mean \pm SEM. (F) Representative H\&E-stained sections of pancreas isolated from mice treated with the cdGMP/CAR T cell scaffold for 1 week or 3 weeks. Scale bars: $100 \mu \mathrm{m}$.

cells in established lesions varied considerably (Figure 10B); this mimics the heterogeneity of antigen expression seen in patients with melanoma (34). We created a CAR directed against GP75 by linking a TA99 hybridoma-derived single-chain antibody to a synthetic receptor skeleton composed of the CD8 hinge, the CD28 transmembrane and signaling domains, and the $\mathrm{CD} 3 \zeta$ signaling domains (Figure 10C). We transduced murine $\mathrm{T}$ cells using recombinant retroviruses expressing these GP75-specific CARs, which enabled them to recognize and lyse B16F10 cells isolated from fresh tumors (Figure 10D). We then treated mice with these GP75 CAR-expressing T cells and/or STING agonists just after surgical resection. As with the pancreatic tumor experiments described above, therapeutics were either directly injected into the resection cavity or launched from implanted biomaterial scaffolds (as illustrated in Figure 10E). We made caliper measurements to monitor the size of relapsing tumors. Results based on the B16F10 melanoma model were similar to those for KPC pancreatic tumors (Figure 9): only scaffold codelivery of cdGMP vaccine adjuvant along with tumor-specific CAR T cells launched synergistic antitumor responses strong enough to eliminate heterogeneous tumors (in 6 of 10 animals; the others showed substantially delayed tumor relapse along with an average improvement in survival of 34 days compared with controls) (Figure 10, E and F). This local treatment led to systemic antitumor immune activity, as long-term survivors always rejected i.v. tumor rechallenge (Figure 10G). As expected, delivering the same dosage of cdGMP/CAR T cells locally via injection into the resection cavity provided only a modest (yet significant) 6-day survival improvement over that of controls.

Thus, our results demonstrate in preclinical models of inoperable pancreatic cancer and incompletely resected melanoma that an appropriately designed biomaterial system can locally release synergistic combinations of anticancer $\mathrm{T}$ cells and adjuvant drugs to transform treated tumors from malignancies into in situ vaccines that trigger host antitumor immunity.

\section{Discussion}

Tumor heterogeneity poses a major obstacle to the success of immunotherapy. Because of this, many cancer treatments use "whole-cell" vaccines prepared from resected tissue that contains the entire antigen spectrum for each patient's primary tumor (3537). By enabling the immune system to react with unique epitopes, these vaccines can evoke reactions against an array of antigens that do not need to be preidentified by the physician. Following tumor dissociation, the purified cancer cells are irradiated and compounded with potent vaccine adjuvants before the patients are immunized, usually via intradermal injections. In an effort to produce more robust immune responses from whole-cell cancer vaccines, clinicians have developed biomaterial-based platforms that can carry patient-specific tumor cells or tumor lysates along with immune-stimulating biomolecules $(38,39)$. Following s.c. implantation, these biomaterials attract circulating APCs, which take up the tumor antigens incorporated in the matrix, and then mature and emigrate into regional lymph nodes where they can prime $\mathrm{T}$ cells that are specific for the cancer. Although these devices may provide significant therapeutic benefits compared with conventional vaccines, they rely on the availability of enough tumor material (which is often limited because of diagnostic needs) to prepare autologous vaccines. Moreover, it may take months of repeated immunizations for an effective response to mature; unfortunately, most malignancies produce substantial clinical deterioration, and often become fatal, within this time frame.

Our laboratory has developed a different approach to achieving rapid tumor destruction that is based on the direct delivery of laboratory-cultivated antitumor lymphocytes. We also designed the polymer implant to convert the tumor bed into a "self" vaccine site, using debris from eradicated tumor cells as the antigen source. Our data establish that, in addition to debulking tumors and increasing antigen release, scaffold-delivered CAR T cells work synergistically with the codelivered vaccine adjuvants to sway the tumor microenvironment from one that is suppressive to one that is permissive.

The mechanisms responsible for activating the host immune system by the corelease of CAR T cells and STING agonists remain to be fully elucidated, but our results indicate that the effects involve more synergy than can be explained by a simple depot effect. Certainly, by simultaneously delivering engineered lymphocytes and vaccine adjuvants from biopolymer implants, the methods described here bring together 3 key components that can propel host antitumor immunity: (a) substantial densities of stimulated CAR T cells that destroy tumor cells; (b) significant amounts of tumor antigens arising from the eradicated cancer cells, which become available to APCs; and (c) high concentrations of stimulants that activate these APCs along with tumor-reactive immune cells to mount a robust anticancer response.

Something that may have improved the success of our treatments is the relatively small tumor burdens in the test systems we used compared with the sizes of the implants. Like the human disease, surgically implanted KPC pancreatic tumors progress rapidly, and this forced us to start therapy early enough to ensure that the animals survived the surgical placement of the scaffolds onto established tumors. Also, it is worth explaining that we resected primary B16 melanomas prior to scaffold implantation to induce the spread of distant metastases (40) (not to reduce tumor load). But it is likely that treating more expansive tumors that are difficult to penetrate will be challenging. We therefore envision that our technology will be most effective when used in combination with 
A

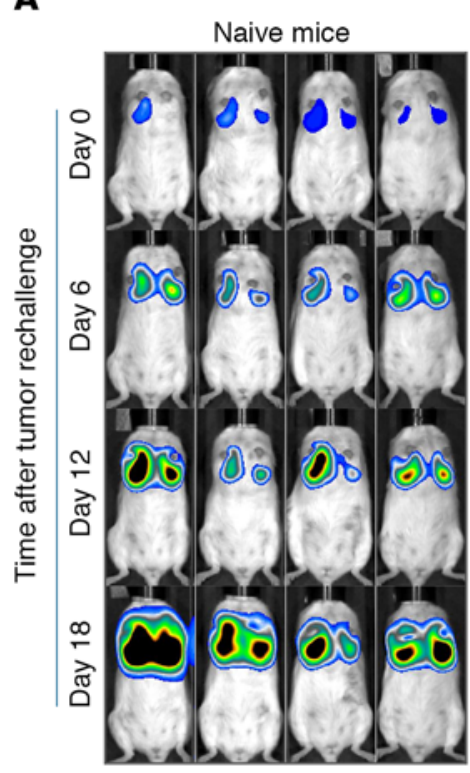

T cell/cdGMP scaffold-treated survivors

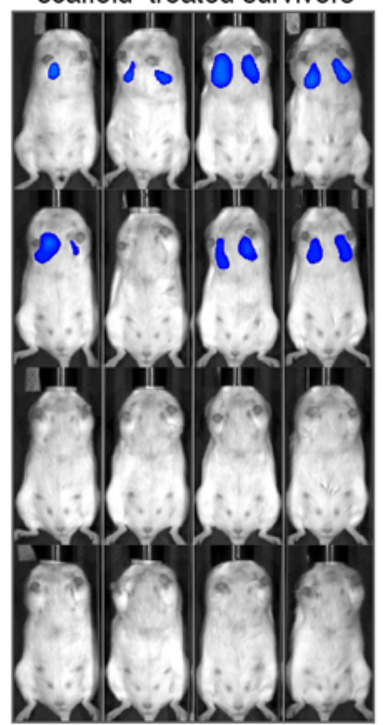

B

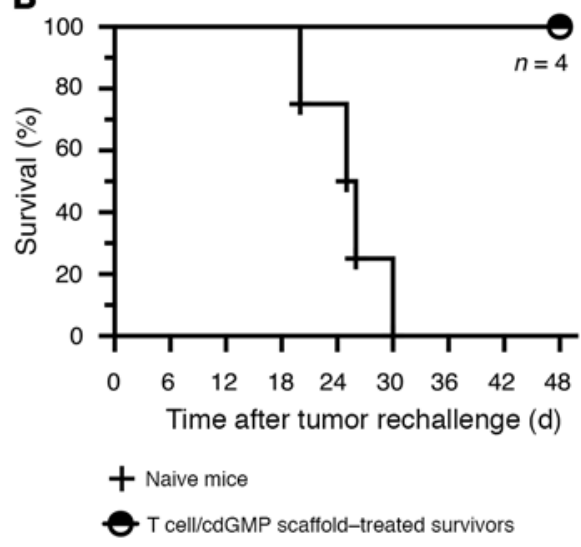

Figure 9. Implants can elicit global antitumor immunity. (A) Serial in vivo bioluminescence imaging of KPC-luc tumor cells injected i.v. into the 4 mice that experienced complete tumor regression, as shown in Figure 8. Age-matched naive mice were used as controls. (B) Kaplan-Meier survival curves. cytoreductive surgery, or as a strategy to reduce the size of inoperable tumors so that patients become eligible for further interventions. In any case, clinical translation will first require optimizing the size, shape, and thickness of the implants, along with doses of $\mathrm{T}$ cells and immune stimulants, for each circumstance to propel penetration by tumor-destroying agents.

For our proof of concept, we chose STING agonists over other immune modulators currently in clinical development, because recent evidence indicates that cyclic dinucleotides targeting this pathway can launch vigorous $\mathrm{T}$ cell responses and thereby cause systemic tumor regression $(7,8)$. To be effective in the interventions currently in use, however, these factors must be repeatedly injected directly into tumor lesions. This limits the therapy to sites that are accessible for daily inoculations (i.e., skin malignancies); as with other powerful immune stimulants, i.v. administration of STING agonists cannot be performed, because it leads to systemic inflammation and toxicity $(12,13)$. By using a biomaterial delivery device that slowly releases the adjuvant directly at the tumor site, we bypass the need for injections: this translates into significant reductions in dosages and minimizes off-target exposure. Besides cyclic dinucleotides, our platform could potentially codeliver (along with CAR T cells) other agonists that stimulate antitumor immune responses, such as anti-checkpoint blockade agents, TLR agonists, indoleamine 2,3-dioxygenase (IDO) inhibitors, TGF- $\beta$ blocking antibodies, adenosine receptor antagonists, or even mixtures of these factors. Our group is particularly interested in testing whether codelivering demethylation drugs (such as azacitidine, which can unmask epigenetically silenced cell-surface proteins and thus make cancer cells more immunogenic [ref. 41]) can further improve the antitumor potential of CAR T cell/STING agonistfunctionalized implants. Given their potential to robustly sensitize tumors to immunotherapy, epigenetic therapies have become the focus of a rapidly emerging area of cancer research (42). However, the significant toxicity these agents produce when applied systemically is a major impediment to their clinical development (43).

Like our selection of the immune stimulant, the choice of the cellular component incorporated into our platform could also be adapted. For example, TCR-engineered lymphocytes have emerged as a useful treatment option for various malignancies $(44,45)$. In this approach, patient-derived cells are programmed to produce engineered TCRs specific for selected tumor antigens. In contrast to the CAR T cells used in our studies (which can only recognize antigens located on the surfaces of tumor cells), TCR gene therapy can target lymphocytes to intracellular tumor antigens, including products of somatic mutations unique to each patient's cancer (46). The question of whether codelivery of adjuvants along with TCR-engineered $\mathrm{T}$ cells can boost the antitumor activity of these T cells (as they do with CAR T cells) must still be evaluated, but the main hurdle for TCR gene therapy is the low (or nonexistent) level of HLA expression by tumor cells, which is a mechanism these cancers exploit to escape immune attacks (47). It is likely that the local release of vaccine adjuvants from implants would upregulate surface expression of HLA molecules by cancer cells and thereby precondition the tumor milieu for the codelivered engineered $\mathrm{T}$ cells that can now recognize and destroy the malignant cells.

In summary, our work demonstrates a new concept in cancer therapy that achieves both rapid tumor clearance and systemic antitumor immunity. The biopolymer scaffold we developed could provide an effective treatment for solid cancers that do not respond well to conventional $\mathrm{T}$ cell therapies, because they are heterogeneous and protected in the microenvironment they create (48). More broadly, we believe that this platform provides a means to shift the focus of cancer treatment from broad-impact chemical and radiation-based interventions to tumor-specific immunotherapeutics by creating a way to safely apply treatments directly to the tumor.

\section{Methods}

\section{Human pancreatic ductal adenocarcinoma}

For confocal imaging of human pancreatic adenocarcinoma (PDA) (Figure 1), fresh tumor tissue was obtained from patients undergoing pancreatic resection for PDA. 
A
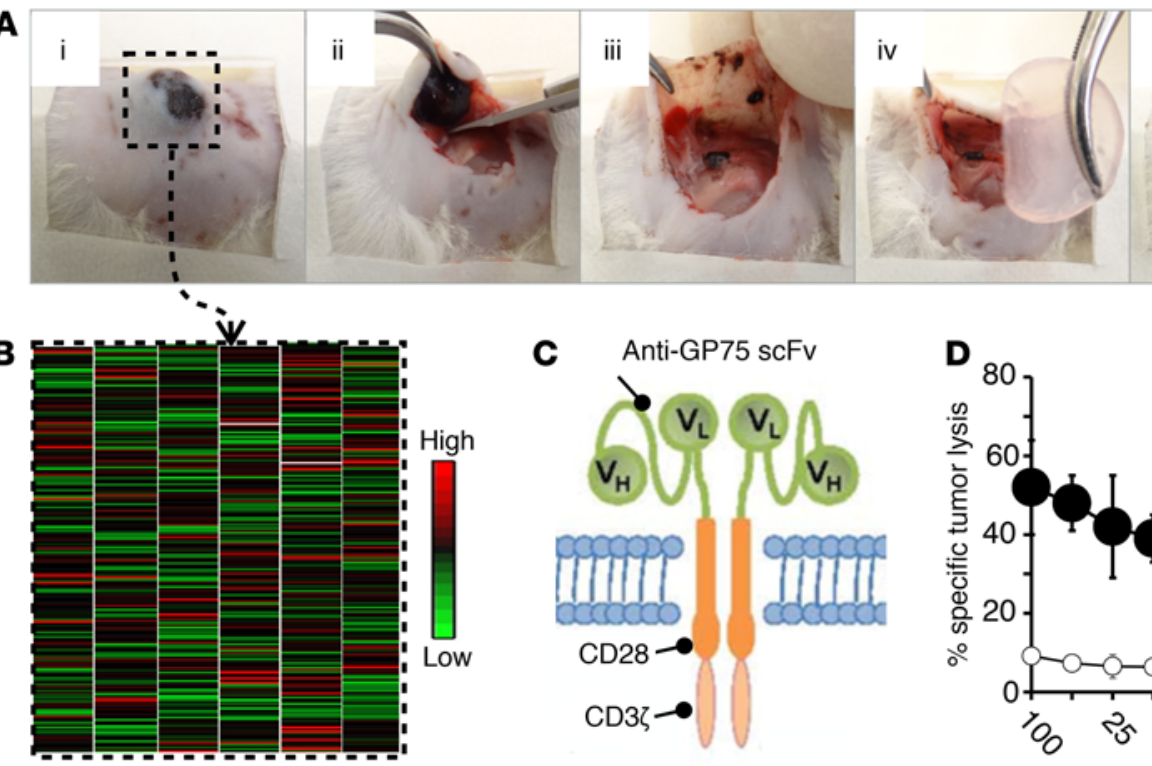

V
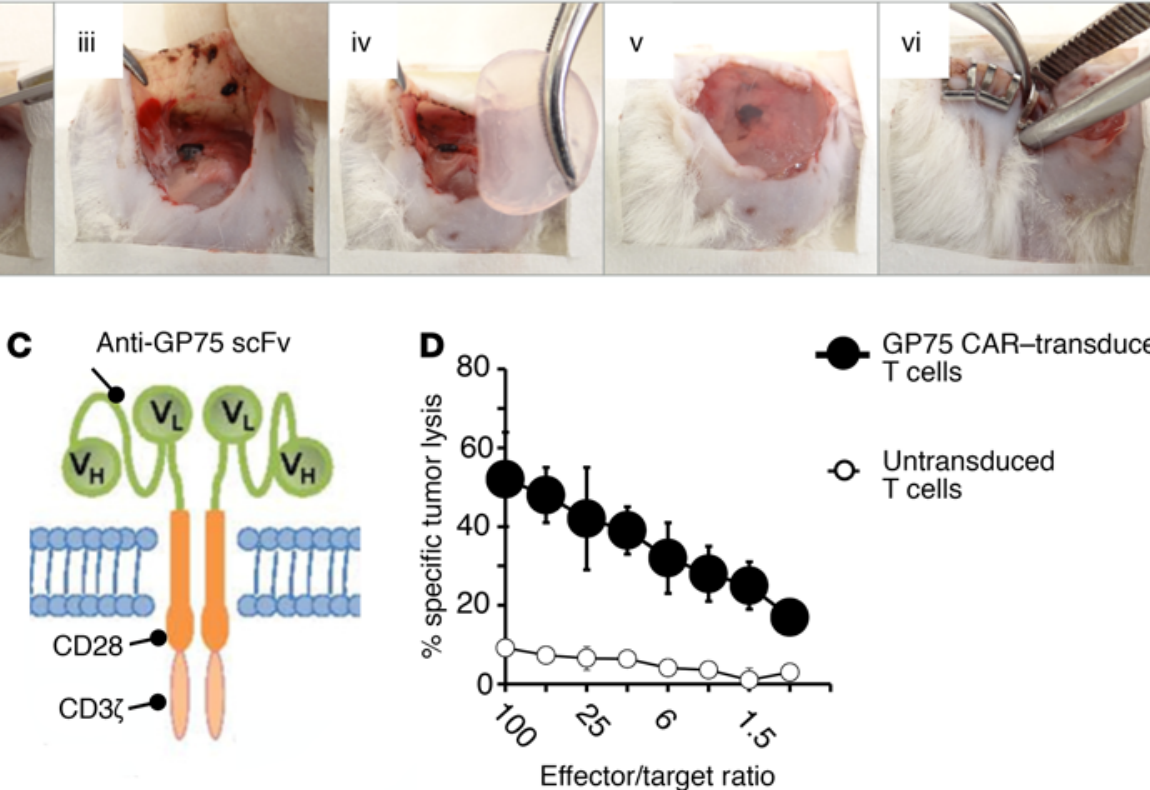

GP75 CAR-transduced T cells

Untransduced T cells
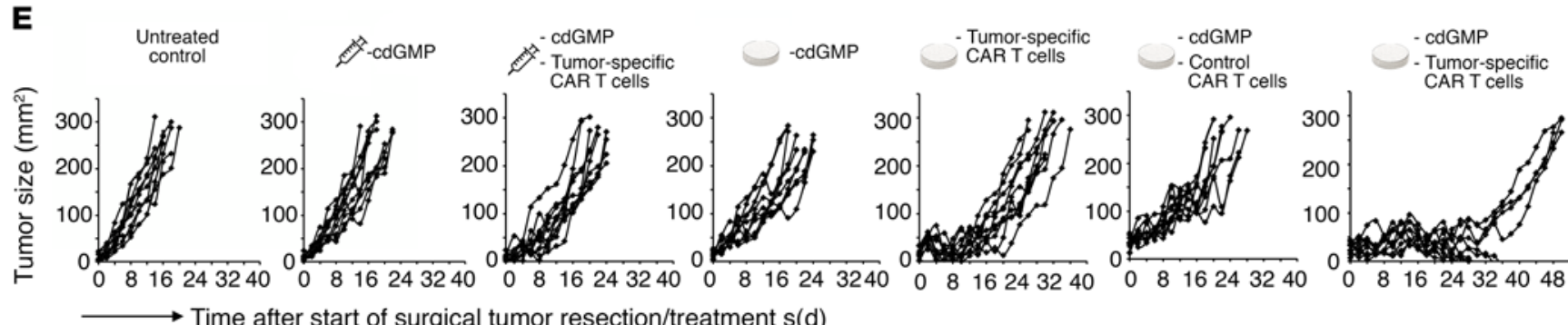

Time after start of surgical tumor resection/treatment $\mathrm{s}(\mathrm{d})$

$\mathbf{F}$

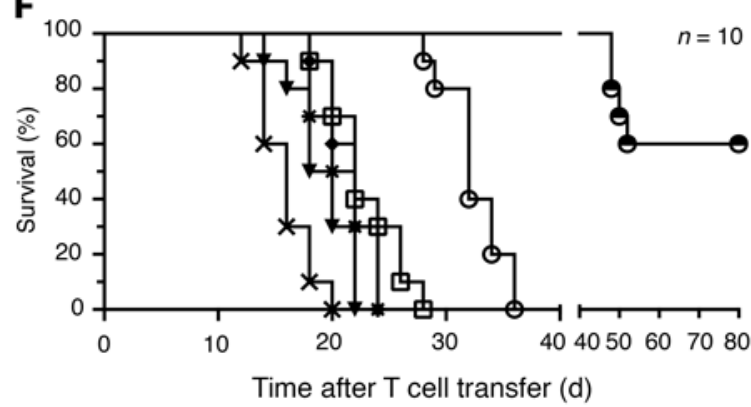

* Untreated control $(\mathrm{ms}=16 \mathrm{~d})$

- Locally injected cdGMP $(\mathrm{ms}=19 \mathrm{~d})] P=0.02^{*}$

$\sim$ Locally injected cdGMP + tumor-specific CAR T cells $(\mathrm{ms}=\mathbf{2 2} \mathrm{d})$

*- Scaffold-delivered cdGMP (ms = $21 \mathrm{~d}$ )

$\ominus$ Scaffold-delivered tumor-specific CAR T cells $(\mathrm{ms}=\mathbf{3 4} \mathrm{d})$

曰 Scaffold-delivered cdGMP + control CAR T cells $(\mathrm{ms}=\mathbf{2 2} \mathrm{d})$

Scaffold-delivered cdGMP + tumor-specific CAR T cells
G

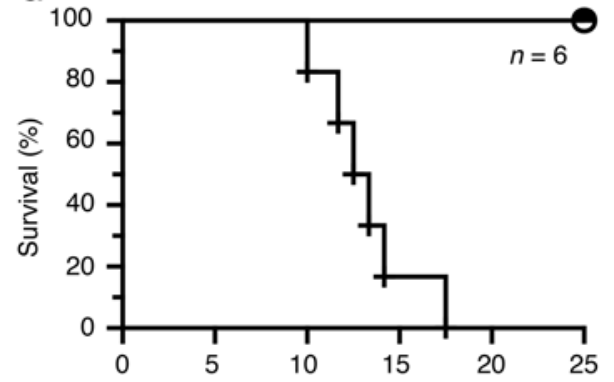

Time after systematic tumor rechallenge (d)

+ Naive mice

T cell/cdGMP scaffold-treated survivors

Figure 10. cdGMP/CAR T cell-functionalized implants can efficiently treat heterogeneous melanoma. (A) Series of images illustrating the codelivery of anticancer T cells and STING agonists via biomaterial carriers to prevent melanoma recurrence after surgery: (i) mouse B16F10 melanoma; (ii) surgical resection; (iii) resection cavity with residual tumor; (iv and v) implantation of biopolymer device; (vi) wound closure. (B) Heatmap depiction of flow cytometric data quantifying the cell-to-cell variability in the expression of GP75 by 10-day-old B16F10 tumors. Colors indicate the relative levels compared with an isotype control, and the results are from 1,800 randomly chosen cells pooled from 5 tumors. (C) Schematic of the anti-GP75 CAR. (D) ${ }^{51} \mathrm{Cr}$ release assay. Each point represents the mean \pm SEM. Data are representative of 4 independent experiments. (E) Progression of B16F10 tumor growth following therapy. Every line represents 1 animal, and each dot reflects the tumor size, as determined by caliper measurements. Data represent 10 animals pooled from 4 independent experiments. (F) Kaplan-Meier survival curves for treated versus control mice. Statistical analysis was performed using the log-rank test, and a $P$ value of less than 0.05 was considered significant. (C) Kaplan-Meier survival curves of the 6 long-term survivors in panels $\mathbf{E}$ and $\mathbf{F}$ and age-matched naive control mice following tail vein injection of $25 \times 10^{4}$ B16F10 tumor cells. Asterisks indicate statistical significance. 


\section{Cell lines}

The murine pancreatic ductal adenocarcinoma (PDA) cell line KPC, a gift of Sunil Hingorani (Fred Hutchinson Cancer Research Center, Seattle, Washington, USA), was derived from spontaneous pancreatic tumors developing in transgenic KPC mice ( $L S L-K r a s^{G 12 D} p 53^{t /++}$ mice) at 17 weeks of age. This cell line was cultured in Iscove's modified Dulbecco's medium (IMDM) with 10\% heat-inactivated FBS, 2 mM Lglutamine, $1.5 \mathrm{~g} / 1$ sodium bicarbonate, $4.5 \mathrm{~g} / 1$ glucose, $10 \mathrm{mM}$ HEPES, $1.0 \mathrm{mM}$ sodium pyruvate, and $0.05 \mathrm{mM}$ 2-mercaptoethanol. B16F10 melanoma cell lines (American Type Culture Collection [ATCC]; catalog CRL-6475) were cultured in complete RPMI 1640 medium with $10 \%$ heat-inactivated FBS, 2 mM L-glutamine, $1.5 \mathrm{~g} / 1$ sodium bicarbonate, $4.5 \mathrm{~g} / 1$ glucose, $10 \mathrm{mM}$ HEPES, $1.0 \mathrm{mM}$ sodium pyruvate, and $0.05 \mathrm{mM}$ 2-mercaptoethanol. Although GP75 occurs on the tumor cell membrane in vivo, surface expression is weak in vitro; therefore, we used B16F10 cells only from fresh tumors for in vitro functional assays. The Phoenix Eco retroviral packaging cell line (Orbigen) was cultured in DMEM containing 10\% FBS, $2 \mathrm{mM}$ glutamate, $100 \mathrm{U} / \mathrm{ml}$ penicillin, and $100 \mu \mathrm{g} / \mathrm{ml}$ streptomycin. For in vivo bioluminescence imaging, the KPC cell line was retrovirally transduced with firefly luciferase (F-luc).

\section{Mice and in vivo tumor models}

Animals were housed in the animal facility of the Fred Hutchinson Cancer Research Center. In our orthotopic mouse model of PDA, 1 $\times 10^{5} \mathrm{KPC}$ tumor cells were surgically implanted into the head of the pancreas of female Albino B6 (C57BL/6J-Tyr<c-2J>) mice (The Jackson Laboratory) and were allowed to establish for 1 week before treatment. Immediately before starting therapy, the KPC tumor burden for each animal was quantified using an in vivo imaging system (IVIS). Only animals with a bioluminescence tumor signal between $2 \times 10^{5}$ and $2 \times$ $10^{6}$ photons $/ \mathrm{s} / \mathrm{cm}^{2}$ were randomly assigned to the various treatment or control groups for our experiments. To differentiate between adoptively transferred and endogenous $\mathrm{T}$ cells in flow cytometry measurements (Figure 5), we generated NKG2D CAR T cells using splenocytes isolated from WT (CD45.2+) C57BL/6 mice. Following gene transfer, T cells were used to treat B6.SJL-Ptprca Pepcb/BoyJ recipient mice (also from The Jackson Laboratory), which, instead of CD45.2, express the panleukocyte marker CD45.1. To directly visualize the location and magnitude of endogenous $\mathrm{T}$ cell activation following different treatment regimens, we bred NFAT-luc-transgenic mice [B6.Cg-Tg(Il2/NFAT-luc) 83Rinc/J; The Jackson Laboratory; stock no. 006098] into an albino B6 $(\mathrm{C} 57 \mathrm{BL} / 6 \mathrm{~J}-\mathrm{Tyr}<\mathrm{c}-2 \mathrm{~J}>$ ) background. In this test system, host T cells emit light following TCR activation, so we orthotopically implanted WT KPC (instead of KPC-luc) tumor cells for these experiments. To establish a preclinical model of incompletely resected melanoma, we injected $1.0 \times$ $10^{6}$ B16F10 cells (in $100 \mu \mathrm{l}$ sterile PBS) subcutaneously into the shaved right flank of anesthetized mice. Once the tumors achieved a size of approximately $150 \mathrm{~mm}^{2}$, we surgically resected them in a way that left behind approximately $1 \%$ to $5 \%$ of the tumor volume.

\section{Retroviral vectors and viral production}

SFG-CBR-luc (which expresses CBR-luc) and SFG-F-luc (which expresses F-luc) vectors were provided by Michel Sadelain (Memorial Sloan Kettering Cancer Center, New York, New York, USA). The retroviral vector $\mathrm{pFb}$-chNKG2D-IRES-Neo, described previously (49), was provided by Charles Sentman (Geisel School of Medicine, Dartmouth College, Lebanon, New Hampshire, USA). NKG2D CAR consists of the full-length mouse NKG2D fused with the cytoplasmic portion of CD36. The GP75-specific CAR consists of a TA99 hybridoma-derived single-chain antibody linked to a synthetic receptor skeleton composed of the CD8 hinge, the CD28 transmembrane and signaling domains, and the signaling domain from $\mathrm{CD} 3 \zeta$. To generate retroviral particles encoding the NKG2D CAR, Phoenix-ECO cells $\left(1.5 \times 10^{6} / 10 \mathrm{~cm}\right.$ culture plate) were transfected overnight with $10 \mu \mathrm{g}$ vector DNA using standard calcium phosphate methods; the following day, they were incubated in $10 \mathrm{ml}$ fresh DMEM for an additional day before the supernatant was filtered (0.45- $\mu \mathrm{m}$ filter; Nalgene) and concentrated 10-fold using Ultracel 100K membranes (EMD Millipore). To transduce mouse T cells with the GP75-specific CAR, we transfected the Platinum-E retroviral packaging cell line (Cell Biolabs) according to the manufacturer's instructions and harvested the retroviral supernatant 48 hours later.

\section{Preparation of tumor-targeting lymphocytes}

To generate pancreatic cancer-specific (NKG2D CAR-transduced) $\mathrm{T}$ cells, spleens from C57BL/6J mice were macerated over a filter and resuspended in ammonium chloride potassium (ACK) lysing buffer (Biosource). Effector CD8 ${ }^{+} \mathrm{T}$ cells were prepared by incubating splenocytes $\left(3 \times 10^{6} / \mathrm{ml}\right)$ in complete RPMI 1640 with $1 \mathrm{ng} / \mathrm{ml} \mathrm{IL-7}$ (PeproTech) and $2 \mu \mathrm{g} / \mathrm{ml}$ concanavalin A (Calbiochem) at $37^{\circ} \mathrm{C}$. Two days later, dead cells were removed by Ficoll gradient separation (GE Healthcare), and then $\mathrm{CD}^{+}$cells were isolated using a mouse CD8 Isolation Kit (STEMCELL Technologies). Retroviral transduction was performed as follows to introduce the NKG2D CAR into T cells: $1 \mathrm{ml}$ of concentrated NKG2D CAR-expressing retrovirus was preloaded into 6-well nontreated dishes coated with RetroNectin (TakiraBio) and incubated at $37^{\circ} \mathrm{C}$ for 1 hour. An equal volume of isolated T cells $(3 \times$ $10^{6}$ cells $/ \mathrm{ml}$ supplemented with $10 \mathrm{ng} / \mathrm{ml}$ mouse IL-2 [mIL-2] [PeproTech]) was added, and the suspension was centrifuged at 2,000 $g$ for 30 minutes. After a 6-hour spinoculation, RPMI containing $10 \mathrm{ng}$ $\mathrm{mIL}-2 / \mathrm{ml}$ was added. Two days after transfection, $0.5 \times 10^{6} / \mathrm{ml}$ to $1 \times$ $10^{6} / \mathrm{ml}$ of the transduced primary $\mathrm{T}$ cells were selected by culturing in RPMI-10 medium containing G418 $(0.5 \mathrm{mg} / \mathrm{ml})$ plus recombinant human IL-2 (25 U/ml) for an additional 3 days. Viable cells were isolated using Histopaque 1083 (Sigma-Aldrich) and expanded for 2 days without $\mathrm{G} 418$ before adoptive transfer. For bioluminescence imaging experiments, the targeted $\mathrm{T}$ cells were genetically tagged with CBR-luc (50). Six hours after spinoculation, $1 \mathrm{ml}$ RPMI containing $50 \mathrm{IU}$ mIL2 was added, and the transduced $\mathrm{T}$ cells were used for experiments the next day. To generate melanoma-specific CAR T cells, we activated mouse T cells with anti-mouse CD3 $(10 \mu \mathrm{g} / \mathrm{ml})$, anti-mouse CD28 $(0.1 \mu \mathrm{g} / \mathrm{ml})$, and $\mathrm{mIL}-2(19 \mathrm{IU} / \mathrm{ml})$ and transduced them 24 hours later with Platinum E-Retroviral supernatant (Cell Biolabs) on RetroNectincoated plates. Following spinoculation $\left(3,000 \mathrm{~g}, 2 \mathrm{~h}, 32^{\circ} \mathrm{C}\right)$, T cells were harvested and resuspended in complete RPMI with IL-2 $(10 \mathrm{IU} / \mathrm{ml})$ and mouse T Activator Beads $\left(0.3 \times 10^{6}\right.$ beads per $1 \times 10^{6} \mathrm{~T}$ cells; Thermo Fisher Scientific). Following a second spinoculation in retroviral supernatant the next day, the cells were cultured for 24 hours in the presence of mouse IL-15 (50 ng/ml) before using them as a tumor therapeutic.

\section{Preparation of stimulatory lipid-coated silica microspheres}

Preparation of a maleimide-functionalized lipid film. Lipid stock solutions were formulated in chloroform. Dioleoyl phosphatidylcholine (DOPC) $(140 \mu \mathrm{l}$ from a 10-mg/ml solution); $30 \mu \mathrm{l}$ 1,2-distearoyl-sn- 
glycero-3-phosphoethanolamine-PEG (DSPE-PEG 2000) maleimide $(5 \mathrm{mg} / \mathrm{ml}) ; 150 \mu \mathrm{l}$ cholesterol (5 mg/ml); and $50 \mu \mathrm{l}$ 18:1 PEG 2000phycoerythrin (PEG 2000-PE) (5 mg/ml), all acquired from Avanti Polar Lipids, were combined to attain a DOPC/DSPE-PEG 2000 maleimide/cholesterol/PEG 2000-PE mass ratio of 55:5:30:10 and 2.5 $\mathrm{mg}$ total lipid. Chloroform was evaporated under a stream of nitrogen, and residual solvent was removed under vacuum overnight.

Amine modification of silica microparticles. Spherical silica gel (500 mg, $15-\mu \mathrm{m}$ particle diameter, 100- $\AA$ pore diameter; Sorbent Technologies) was suspended in $4 \mathrm{ml}$ of $25 \% 3$-[2-(2-aminoethylamino) ethylamino]propyltrimethoxysilane (AEPTMS) in ethanol and then mixed gently at room temperature for 5 hours. Unreacted AEPTMS was removed by centrifugation (1,000 $\mathrm{g}$ for $2 \mathrm{~min}$ ) and decantation of the supernatant. The amine-modified silica was washed with ethanol $(4 \times 2 \mathrm{ml})$ and then air dried for 2 days.

Loading of STING agonist into mesoporous silica microparticles. A $100-\mathrm{mg} / \mathrm{ml}$ suspension of amine-modified silica was prepared in PBS at $\mathrm{pH} 7.2$, then $360 \mu \mathrm{l}$ of this was combined with $500 \mu \mathrm{l}$ cdGMP ( $2 \mathrm{mg} / \mathrm{ml}$ in PBS; InvivoGen). The solution was gently vortexed for 1 hour, followed by dilution with $400 \mu \mathrm{l}$ PBS.

Lipid adsorption on silica. The $\mathrm{SiO}_{2} / \mathrm{cdGMP}$ suspension $(400 \mu \mathrm{l})$ was added to a 2.5-mg batch of lipid film and vortexed for $15 \mathrm{sec}-$ onds at 10-minute intervals for a total of 1 hour. The particles were pelleted at 3,500 $\mathrm{g}$ for 2 minutes, washed with PBS $(2 \times 1 \mathrm{ml})$, and then resuspended in $250 \mu \mathrm{lBS}$

\section{Antibody conjugation to lipid-coated microparticles}

The following antibodies were purchased from BioXcell: InVivoMAb anti-mouse CD3, clone 17A2 (catalog BE0002); InVivoMAb anti-mouse CD28, clone 37.51 (catalog BE0015-1); and InVivoMAb anti-mouse 4-1BB (CD137), clone LOB12.3 (catalog BE0169). The hinge region disulfide bonds of anti-mouse CD3, CD28, and CD137 antibodies were selectively reduced with DTT as previously described (51). After DTT was removed with a desalting column, these mildly reduced antibodies (anti-CD3: $200 \mu \mathrm{g}$; anti-CD28 and CD137: $400 \mu \mathrm{g}$ ) were added to 250 $\mu \mathrm{l}$ maleimide-functionalized particles, vortexed briefly before rotation for 2 hours, and centrifuged at 3,500 $g$ for 2 minutes. The pellet was washed with PBS $(2 \times 1 \mathrm{ml})$ and then suspended in $125 \mu \mathrm{PBS}$.

\section{Scaffold fabrication}

We produced the alginate scaffolds from high-molecular-weight $(\sim 250$ $\mathrm{kDa}$ ) ultrapure sodium alginate powder (Novamatrix Pronova UP MVG alginate) enriched in $\mathrm{G}$ blocks ( $\geq 60 \%$ ) after it was oxidized with sodium periodate to create hydrolytically labile bonds, as previously described (52). Briefly, $200 \mu \mathrm{l}$ of $0.25 \%$ sodium periodate was added dropwise to $10 \mathrm{ml}$ aqueous $1 \%$ alginate and stirred in the dark at $25^{\circ} \mathrm{C}$ for 5 hours before the reaction was quenched by adding equimolar ethylene glycol for 30 minutes. The sample was dialyzed against deionized water for 3 days using membranes with a 3,500-molecular-weight cutoff and then lyophilized. The oxidized alginate was reconstituted in 2-(N-morpholino)ethane-sulfonic acid (MES) buffer (0.1 M MES and $0.3 \mathrm{M} \mathrm{NaCl}, \mathrm{pH}$ 6.5) and covalently conjugated to the collagen-mimetic peptide GFOGER (53) (obtained from the MIT Biopolymers facility) using carbodiimide chemistry (54): sulfo- $N$-hydroxysulfosuccinimide (Sulfo-NHS), 1-ethyl-3-(3-dimethylaminopropyl)carbodiimide hydrochloride (EDC) (both from Thermo Fisher Scientific), and the GFOGER peptide were added sequentially, and 24 hours later the solution was again dialyzed (20-kDa molecular weight cutoff [MWCO] dialysis membrane; Thermo Fisher Scientific) and lyophilized.

To make scaffolds, the alginate stock was reconstituted to $7 \mathrm{ml}$ of a $2 \% \mathrm{w} / \mathrm{v}$ solution in PBS and warmed to $55^{\circ} \mathrm{C}$ before mixing with $7 \times 10^{6}$ stimulatory microspheres in aqueous suspension. Mild cross-linking was initiated by adding $1.4 \mathrm{ml}$ of a $0.1 \%(\mathrm{w} / \mathrm{v})$ calcium chloride solution while vortexing, and then $700 \mu \mathrm{l}$ was immediately transferred into 15-mm round, Teflon-coated molds to form 2-mm-thick scaffolds. These were frozen at $-78^{\circ} \mathrm{C}$ and lyophilized to yield porous matrices, which were stored at $4^{\circ} \mathrm{C}$ in a desiccator.

\section{T cell seeding onto scaffolds}

Mouse T cells genetically engineered to express NKG2D-CAR or antiGP75-CAR were washed twice in PBS and resuspended in nonsupplemented RPMI medium at a concentration of $14 \times 10^{6}$ cells $/ \mathrm{ml}$. After adding 5\% AlgiMatrix Firming Buffer (Invitrogen, Thermo Fisher Scientific), $500 \mu$ lof this cell suspension was immediately inoculated on top of each lyophilized scaffold in a 24-well tissue culture plate. Cells were allowed to infuse into these matrices for 30 minutes on ice before implantation into the peritoneal or tumor resection cavity.

\section{Cytotoxicity assays}

We measured the in vitro cytotoxic activity of $\mathrm{T}$ cells using standard ${ }^{51} \mathrm{Cr}$ release assays as described elsewhere (55). Briefly, KPC or B16F10 cells were labeled with ${ }^{51} \mathrm{Cr}$ for 1 hour at $37^{\circ} \mathrm{C}$, washed with RPMI containing $10 \% \mathrm{FCS}$, and resuspended in the same medium at a concentration of $1 \times 10^{5}$ tumor cells $/ \mathrm{ml}$. T cells were added to the suspensions at varying effector-to-target cell ratios in 96-well plates (final volume, $200 \mu \mathrm{l}$ ) and incubated for 4 hours at $37^{\circ} \mathrm{C}$, then $30 \mu \mathrm{l}$ of supernatant from each well was transferred into Lumaplate-96 microplates (Packard BioScience) for analysis with a Top Count NXT microplate scintillation counter (Packard BioScience).

\section{In vivo bioluminescence and imaging}

We used D-luciferin (Xenogen) in PBS $(15 \mathrm{mg} / \mathrm{ml})$ as a substrate for F-luc (imaging of KPC pancreatic tumors) and CBR-luc (50) (T cell imaging). Bioluminescence images were collected with a Xenogen IVIS Spectrum Imaging System. Living Image software, version 4.3.1 (Xenogen), was used to acquire (and later quantitate) the data 10 minutes after i.p. injection of D-luciferin into animals anesthetized with 150 $\mathrm{mg} / \mathrm{kg}$ of $2 \%$ isoflurane (Forane; Baxter Healthcare). Acquisition times ranged from 10 seconds to 5 minutes. Only acquisitions with unsaturated signals were included in the analysis. To correct for background bioluminescence, we subtracted signals acquired from healthy WT mice (injected with D-luciferin) from the region of interest (ROI) measured.

\section{Toxicity studies}

To measure potential in vivo toxicities of cdGMP/CAR T cellfunctionalized implants, we established KPC pancreatic tumors in 4to 6-week-old female C57BL/6 mice, then implanted scaffolds containing either NKG2D-CAR ${ }^{+} \mathrm{T}$ cells, cdGMP, or both (5 mice/group). The control animals received no treatment. Seven days after scaffold implantation, body weights were measured, mice were anesthetized, and blood was collected by cardiac puncture into microcontainers containing EDTA. Analysis (conducted at the Phoenix Central Laboratory, Mukitteo, Washington, USA) consisted of a complete blood count, which included a white cell count with differential, as well as red cell, 
hemoglobin, hematocrit, and platelet counts. Blood was also collected into serum separator tubes and sent to the same laboratory for analysis of amylase and lipase serum levels. Animals were then euthanized with carbon dioxide to retrieve their pancreas. The excised organs were washed with deionized water and fixed with $4 \%$ paraformaldehyde solution. The tissues were processed in a routine fashion, and sections were stained with H\&E. All specimens were examined in a blinded fashion by Smitha Pillai, a board-certified staff pathologist.

\section{Flow cytometry}

Anti-recombinant annexin V (used to quantify apoptotic cells) and the following antibodies (used with the BD FACSCanto Flow Cytometer) were purchased from eBioscience: anti-NKG2D (catalog 130312; clone CX5); anti-CD8 (catalog 100738; clone 53-6.7); anti-CD11b (catalog 101226; clone M1/70); anti-CD11c (catalog 117330; clone N418); antiCD86 (catalog 105039; clone GL-1); anti-MHC II (catalog 12-5321-81; clone M5/114.15.2); and anti-CD45.1 (catalog 45-0453-82; clone A20).

$\mathrm{H}-2 \mathrm{D}^{\mathrm{b}}$ LCMV GP33 Tetramer-KAVYNFATC was synthesized in the Immune Monitoring Core Facility at the Fred Hutchinson Cancer Research Center.

\section{Confocal microscopy}

Sections (4- $\mu \mathrm{m}$-thick) of formalin-fixed human PDA were deparaffinized in xylene and rehydrated through a graded ethanol series before heat-mediated antigen retrieval in $10 \mathrm{mM}$ sodium citrate buffer $(\mathrm{pH}$ 6.0). Following PBS washing and treatment with $0.1 \%$ Triton $\mathrm{X}-100$ for 15 minutes, the cells were blocked with $10 \%$ normal goat serum in $\mathrm{PBS}$ for 30 minutes at room temperature, then incubated at $4^{\circ} \mathrm{C}$ overnight with the following antibodies (as indicated in the figure legends): Alexa Fluor 594 anti-human CD326 (EpCAM) (324228, clone 9C4; BioLegend); FITC pan-cytokeratin (ab78478, clone C-11; Abcam); and rabbit anti-mesothelin (ab93620, clone SP74; Abcam). Samples were washed and then incubated with Alexa Fluor 647-conjugated antirabbit (4414S; Cell Signaling Technology) as appropriate for 1 hour at room temperature. The samples were then washed with PBS twice and counterstained with DAPI. Confocal microscopy was performed with a Leica SP8X confocal microscope (Leica Microsystems), and images were analyzed using Fiji Image (NIH).

\section{Statistics}

The statistical significance of differences measured in T cell migration parameters was calculated using 1-way ANOVA, followed by Dunnett's comparison test. Pairwise differences in bioluminescent tumor and $\mathrm{T}$ cell signals were analyzed at selected time points using the Wilcoxon rank-sum test. A $P$ value of less than 0.05 was considered significant. Survival data were characterized using the log-rank test. All statistical analyses were performed using GraphPad Prism, version 6.0 (GraphPad Software).

\section{Study approval}

Experiments and handling of mice was conducted following federal, state, and local guidelines under an IACUC protocol and with approval of the IACUC of the Fred Hutchinson Cancer Research Center. All patients provided informed consent under a research protocol approved by the Cancer Consortium IRB at the Fred Hutchinson Cancer Research Center.

\section{Author contributions}

TTS performed in vivo T cell-priming studies; HFM phenotyped KPC tumors following therapy; SBS prepared scaffolds and microparticles; CFO helped develop the retroviral vector PMP71 encoding the GP75-specific CAR; AGD helped with adoptive T cell experiments; XJ performed the confocal microscopy; VGP provided human pancreatic tumor samples; SPSP performed serum analyses and histopathology; KDW helped develop the retroviral vector pMP71 encoding the GP75-specific CAR; and MTS designed the study, performed experiments, analyzed and interpreted data, and wrote the manuscript.

\section{Acknowledgments}

We thank Sunil Hingorani (Fred Hutchinson Cancer Research Center) for the KPC cell line. This work was supported in part by the Fred Hutchinson Cancer Research Center's Immunotherapy Initiative with funds provided by the Bezos Family Foundation; the National Cancer Institute (NCI), NIH (RO1 CA181413); and a Solid Tumor Translational Research (STTR) Translational Research Grant.

Address correspondence to: Matthias T. Stephan, Fred Hutchinson Cancer Research Center, 1100 Fairview Avenue North, Thomas Research Building, Mail Stop D3-100, Seattle, Washington 98109-1024, USA. Phone: 617.667.6677; E-mail: mstephan@ fredhutch.org.

CFO's present address is: Gilead, Oceanside, California, USA.

AGD's present address is: Centre for Infection and Immunity, Queen's University, Belfast, Ireland.
1. Mellman I, Coukos G, Dranoff G. Cancer immunotherapy comes of age. Nature. 2011;480(7378):480-489.

2. Sheikh NA, et al. Sipuleucel-T immune parameters correlate with survival: an analysis of the randomized phase 3 clinical trials in men with castration-resistant prostate cancer. Cancer Immunol Immunother. 2013;62(1):137-147.

3. Kantoff PW, et al. Overall survival analysis of a phase II randomized controlled trial of a Poxviralbased PSA-targeted immunotherapy in metastatic castration-resistant prostate cancer. JClin Oncol. 2010;28(7):1099-1105.

4. Amato RJ, Stepankiw M. Clinical Efficacy of Tro-
Vax in the Treatment of Progressive Castrationresistant Prostate Cancer. Clin Med Insights Oncol. 2012;6:67-73.

5. Madan RA, et al. Ipilimumab and a poxviral vaccine targeting prostate-specific antigen in metastatic castration-resistant prostate cancer: a phase 1 dose-escalation trial. Lancet Oncol. 2012;13(5):501-508.

6. Barber GN. STING-dependent cytosolic DNA sensing pathways. Trends Immunol. 2014;35(2):88-93.

7. Fu J, et al. STING agonist formulated cancer vaccines can cure established tumors resistant to PD-1 blockade. Sci Transl Med. 2015;7(283):283ra52.

8. Corrales L, et al. Direct Activation of STING in the Tumor Microenvironment Leads to Potent and Systemic Tumor Regression and Immunity. Cell Rep. 2015;11(7):1018-1030.

9. Harrison LI, Astry C, Kumar S, Yunis C. Pharmacokinetics of 852A, an imidazoquinoline Toll-like receptor 7-specific agonist, following intravenous, subcutaneous, and oral administrations in humans. J Clin Pharmacol. 2007;47(8):962-969.

10. Walder $\mathrm{P}$, et al. Pharmacokinetic profile of the immunomodulating compound adamantylamide dipeptide (AdDP), a muramyl dipeptide deriva- 
tive in mice. Immunopharmacol Immunotoxicol. 1991;13(1-2):101-119.

11. Kulkarni RR, et al. Activation of the RIG-I pathway during influenza vaccination enhances the germinal center reaction, promotes $\mathrm{T}$ follicular helper cell induction, and provides a dose-sparing effect and protective immunity.J Virol. 2014;88(24):13990-14001.

12. Hanson MC, et al. Nanoparticulate STING agonists are potent lymph node-targeted vaccine adjuvants. J Clin Invest. 2015;125(6):2532-2546.

13. Gray PM, et al. Evidence for cyclic diguanylate as a vaccine adjuvant with novel immunostimulatory activities. Cell Immunol. 2012;278(1-2):113-119.

14. Sadelain M, Brentjens R, Rivière I. The basic principles of chimeric antigen receptor design. Cancer Discov. 2013;3(4):388-398.

15. Barrett DM, Grupp SA, June CH. Chimeric Antigen Receptor- and TCR-Modified T Cells Enter Main Street and Wall Street. JImmunol. 2015;195(3):755-761.

16. Grupp SA, et al. Chimeric antigen receptormodified $\mathrm{T}$ cells for acute lymphoid leukemia. N EnglJ Med. 2013;368(16):1509-1518.

17. Brentjens RJ, et al. CD19-targeted T cells rapidly induce molecular remissions in adults with chemotherapy-refractory acute lymphoblastic leukemia. Sci Transl Med. 2013;5(177):177ra38.

18. Motz GT, Coukos G. Deciphering and reversing tumor immune suppression. Immunity. 2013;39(1):61-73.

19. John LB, Kershaw MH, Darcy PK. Blockade of PD-1 immunosuppression boosts CAR T-cell therapy. Oncoimmunology. 2013;2(10):e26286.

20. Johnson DB, et al. Severe cutaneous and neurologic toxicity in melanoma patients during vemurafenib administration following anti-PD-1 therapy. Cancer Immunol Res. 2013;1(6):373-377.

21. Läubli H, Balmelli C, Bossard M, Pfister O, Glatz K, Zippelius A. Acute heart failure due to autoimmune myocarditis under pembrolizumab treatment for metastatic melanoma. JImmunother Cancer. 2015;3:11.

22. Postow MA. Managing immune checkpointblocking antibody side effects. Am Soc Clin Oncol Educ Book. 2015;76-83.

23. Parkhurst MR, et al. T Cells Targeting Carcinoembryonic Antigen Can Mediate Regression of Metastatic Colorectal Cancer but Induce Severe Transient Colitis. Mol Ther. 2011;19(3):620-626.

24. Morgan RA, Yang JC, Kitano M, Dudley ME, Laurencot CM, Rosenberg SA. Case report of a serious adverse event following the administration of $\mathrm{T}$ cells transduced with a chimeric antigen receptor recognizing ERBB2. Mol Ther. 2010;18(4):843-851.

25. Stephan SB, Taber AM, Jileaeva I, Pegues EP, Sentman CL, Stephan MT. Biopolymer implants enhance the efficacy of adoptive T-cell therapy. Nat Biotechnol. 2015;33(1):97-101.

26. Hingorani SR, et al. Trp53R172H and KrasG12D cooperate to promote chromosomal instability and widely metastatic pancreatic ductal adenocarcinoma in mice. Cancer Cell. 2005;7(5):469-483.

27. Sentman CL, Meehan KR. NKG2D CARs as cell therapy for cancer. Cancer J. 2014;20(2):156-159.

28. Baldwin AD, Kiick KL. Polysaccharide-modified synthetic polymeric biomaterials. Biopolymers. 2010;94(1):128-140.

29. Macian F. NFAT proteins: key regulators of T-cell development and function. Nat Rev Immunol. 2005;5(6):472-484

30. Fox JG, Barthold S, Davisson M, Newcomer CE, Quimby FW, Smith A, eds. The Mouse in Biomedical Research, Volume 3, Second Edition: Normative Biology, Husbandry, and Models. Cambridge, MA: Academica Press: 2007.

31. Ghanem G, Fabrice J. Tyrosinase related protein 1 (TYRP1/gp75) in human cutaneous melanoma. Mol Oncol. 2011;5(2):150-155.

32. Boross $\mathrm{P}$, et al. Anti-tumor activity of human IgG1 anti-gp75 TA99 mAb against B16F10 melanoma in human FcgammaRI transgenic mice. Immunol Lett. 2014;160(2):151-157.

33. Albanesi M, et al. Cutting edge: Fc $\gamma$ RIII (CD16) and Fc $\gamma \mathrm{RI}$ (CD64) are responsible for antiglycoprotein 75 monoclonal antibody TA99 therapy for experimental metastatic B16 melanoma. JImmunol. 2012;189(12):5513-5517.

34. Bolander A, et al. The protein expression of TRP-1 and galectin- 1 in cutaneous malignant melanomas Cancer Genomics Proteomics. 2008;5(6):293-300.

35. Clavreul A, et al. Autologous tumor cell vaccination plus infusion of GM-CSF by a programmable pump in the treatment of recurrent malignant gliomas. JClin Neurosci. 2010;17(7):842-848.

36. Muragaki Y, et al. Phase I/IIa trial of autologous formalin-fixed tumor vaccine concomitant with fractionated radiotherapy for newly diagnosed glioblastoma. Clinical article. J Neurosurg. 2011;115(2):248-255.

37. Zhou F, et al. InCVAX--a novel strategy for treatment of late-stage, metastatic cancers through photoimmunotherapy induced tumor-specific immunity. Cancer Lett. 2015;359(2):169-177.

38. Bencherif SA, et al. Injectable cryogel-based whole-cell cancer vaccines. Nat Commun. 2015;6:7556.

39. Kim J, et al. Injectable, spontaneously assembling, inorganic scaffolds modulate immune cells in vivo and increase vaccine efficacy. Nat Biotechnol. 2015;33(1):64-72.

40. Otsubo D, et al. Early-phase Treatment by Lowdose 5-Fluorouracil or Primary Tumor Resection Inhibits MDSC-mediated Lung Metastasis Formation. Anticancer Res. 2015;35(8):4425-4431.

41. Gang AO, et al. 5-Azacytidine treatment sensi- tizes tumor cells to T-cell mediated cytotoxicity and modulates NK cells in patients with myeloid malignancies. Blood Cancer J. 2014;4:e197.

42. Dear AE. Epigenetic Modulators and the New Immunotherapies. $N$ Engl JMed. 2016;374(7):684-686.

43. Héninger E, Krueger TE, Lang JM. Augmenting antitumor immune responses with epigenetic modifying agents. Front Immunol. 2015;6:29.

44. Draper LM, et al. Targeting of HPV-16+ Epithelial Cancer Cells by TCR Gene Engineered T Cells Directed against E6. Clin Cancer Res. 2015;21(19):4431-4439.

45. Robbins PF, et al. A pilot trial using lymphocytes genetically engineered with an NY-ESO-1reactive T-cell receptor: long-term follow-up and correlates with response. Clin Cancer Res. 2015;21(5):1019-1027.

46. Parkhurst MR, et al. Isolation of $\mathrm{T}$ cell receptors specifically reactive with mutated tumor associated antigens from tumor infiltrating lymphocytes based on CD137 expression [published online ahead of print November 8, 2016]. Clin Cancer Res. https://doi.org/10.1158/1078-0432. CCR-16-2680.

47. Thor Straten P, Garrido F. Targetless T cells in cancer immunotherapy. J Immunother Cancer. 2016;4:23.

48. Meacham CE, Morrison SJ. Tumour heterogeneity and cancer cell plasticity. Nature. 2013;501(7467):328-337.

49. Zhang T, Lemoi BA, Sentman CL. Chimeric NK-receptor-bearing $\mathrm{T}$ cells mediate antitumor immunotherapy. Blood. 2005;106(5):1544-1551.

50. Dobrenkov K, et al. Monitoring the efficacy of adoptively transferred prostate cancer-targeted human T lymphocytes with PET and bioluminescence imaging. J Nucl Med. 2008;49(7):1162-1170.

51. Kwong B, Gai SA, Elkhader J, Wittrup KD, Irvine DJ. Localized immunotherapy via liposomeanchored Anti-CD137 + IL-2 prevents lethal toxicity and elicits local and systemic antitumor immunity. Cancer Res. 2013;73(5):1547-1558.

52. Boontheekul T, Kong HJ, Mooney DJ. Controlling alginate gel degradation utilizing partial oxidation and bimodal molecular weight distribution. Biomaterials. 2005;26(15):2455-2465.

53. Wojtowicz AM, et al. Coating of biomaterial scaffolds with the collagen-mimetic peptide GFOGER for bone defect repair. Biomaterials. 2010;31(9):2574-2582.

54. Rowley JA, Mooney DJ. Alginate type and RGD density control myoblast phenotype. J Biomed Mater Res. 2002;60(2):217-223.

55. Erskine CL, Henle AM, Knutson KL. Determining optimal cytotoxic activity of human Her2neu specific $\mathrm{CD} 8 \mathrm{~T}$ cells by comparing the $\mathrm{Cr} 51$ release assay to the xCELLigence system. J Vis Exp. 2012;(66):e3683. 